\title{
Anisotropy of Eddy Variability in the Global Ocean
}

\author{
K. D. Stewarta,b,c,*, P. Spence ${ }^{\mathrm{b}, \mathrm{c}}$, S. Waterman ${ }^{\mathrm{b}, \mathrm{c}, \mathrm{d}}$, J. Le Sommer ${ }^{\mathrm{e}}$, J.-M. \\ Molines $^{\mathrm{e}}$, J. M. Lilly ${ }^{\mathrm{f}}$, M. H. England ${ }^{\mathrm{b}, \mathrm{c}}$ \\ ${ }^{a}$ Research School of Earth Sciences, Australian National University, Australia \\ ${ }^{b}$ Climate Change Research Centre, University of New South Wales, Australia \\ ${ }^{c}$ Australian Research Council Centre of Excellence for Climate System Science \\ ${ }^{d}$ Department of Earth, Ocean $\&$ Atmospheric Sciences, University of British Columbia, \\ Canada \\ ${ }^{e}$ Laboratoire de Glaciologie et Géophysique de l'Environnement (LGGE), \\ CNRS/Université de Grenoble, France \\ ${ }^{f}$ NorthWest Research Associates, Washington, USA
}

\begin{abstract}
The anisotropy of eddy variability in the global ocean is examined in geostrophic surface velocities derived from satellite observations and in the horizontal velocities of a $1 / 12^{\circ}$ global ocean model. Eddy anisotropy is of oceanographic interest as it is through anisotropic velocity fluctuations that the eddy and mean-flow fields interact dynamically. This study is timely because improved observational estimates of eddy anisotropy will soon be available with Surface Water and Ocean Topography (SWOT) altimetry data. We find there to be good agreement between the characteristics and distributions of eddy anisotropy from the present satellite observations and model ocean surface. In the model, eddy anisotropy is found to have significant vertical structure and is largest close to the ocean bottom, where the anisotropy aligns with the underlying isobaths. The highly anisotropic bottom signal is almost entirely contained in the barotropic variability. Upper-ocean variability is predominantly baroclinic and the alignment is less sensitive to the underlying bathymetry. These finding offer guidance for introducing a parameterization of eddy feedbacks, based on the eddy kinetic energy and underlying bathymetry, to operate on the barotropic flow and better account for the effects of barotropic Reynolds stresses unresolved in coarse-resolution ocean models.

Keywords: ocean eddies, barotropic/baroclinic variability, bathymetry-induced variability, variance ellipses
\end{abstract}

${ }^{*}$ Corresp. Author. email: kial stewart@anu.edu.au Preprint submitted to Ocean Modelling

September 11, 2015 


\section{Introduction}

Variability, particularly at the mesoscale $(10-100 \mathrm{~km})$, accounts for the majority of the ocean's kinetic energy (e.g., Ducet et al., 2000; Wunsch, 2007; McWilliams , 2008) and plays a fundamental role in setting the ocean state and circulation (e.g., Hallberg $\&$ Gnanadesikan, 2006; Waterman et al. , 2011; Fox-Kemper et al. , 2013), including air-sea $\mathrm{CO}_{2}$ fluxes (Dufour et al. , 2013). The fluctuating, or eddy, component of the velocity field interacts with the time-mean flow only through the anisotropic or directional portion of the variability (Hoskins et al., 1983; see also Marshall et al., 2012, Waterman \&6 Hoskins, 2013 and Waterman $\&$ Lilly, 2015), and as such, insight into the dynamical feedbacks between the eddy and mean-flow fields of the global oceans can be gained by quantifying and characterizing the magnitude and distribution of eddy anisotropy. Knowledge of eddy anisotropy is especially useful for improving parameterizations of unresolved variability in coarseresolution ocean models (e.g., Eden, 2010), and defining criteria for where to prescribe these parameterizations (Hallberg, 2013).

Quantifying the anisotropy of ocean variability and understanding its mean-flow feedback requires an appropriate methodology. Typically this involves a Reynolds decomposition of ocean velocity and tracer fields, partitioning them into the time-mean and time-varying (eddy) components, and then identifying the conditions where these two components interact (e.g., Marshall, 1984; Plumb , 1986; Gent \& McWilliams , 1996). A convenient framework are variance ellipses (see, for example, Preisendorfer, 1988) which provide a visual representation of eddy momentum fluxes. Variance ellipses characterize the horizontal velocity variances and covariance, thereby capturing the geometry of the fluctuating portion of the horizontal velocity and its associated horizontal momentum fluxes in terms of the eddy kinetic energy (which sets the ellipse amplitude), degree of anisotropy (which sets the ellipse eccentricity) and orientation (direction of the principle axis along which the anisotropy is oriented). Knowledge of the degree of anisotropy and its orientation are important because eddy-mean-flow interactions are sensitive to the magnitude of the anisotropic eddy kinetic energy and to the extent that the eddy anisotropy orientation departs from the direction of the mean-flow shear. Variance ellipses have previously been used in atmospheric research (e.g., Hoskins et al., 1983), in the analysis of idealized ocean models (e.g., 
Marshall et al., 2012; Waterman $\mathcal{E}$ Hoskins , 2013) and observational studies (e.g., Morrow et al., 1994; Stanton 83 Morris, 2004) to represent the characteristics of the variability and the interaction between the eddying and mean flows. Improved observational estimates for variance ellipses and eddy anisotropy of the ocean surface will soon be available through Surface Water and Ocean Topography (SWOT) altimetry data (e.g., Durand et al., 2010; Fu et al. , 2010), increasing the need to appreciate the representation of these metrics in present observations and modelling efforts.

This paper examines the characteristics and global distributions of the anisotropy of ocean eddy variability from satellite observations and a $1 / 12^{\circ}$ global ocean circulation model. It is found that eddy anisotropy is rich in both horizontal and vertical structure. In the model, eddy anisotropy increases towards the ocean bottom, where the highly anisotropic bottom variability is aligned with the underlying isobaths. Decomposing the horizontal flow into its barotropic and baroclinic components reveals that the anisotropy of the bottom variability is almost entirely contained in the barotropic flow, while the relatively isotropic upper-ocean variability is predominantly baroclinic. This offers guidance for the development of a parameterization for the effects of unresolved barotropic variability that is based on the shape of the underlying bathymetry and operates only on the barotropic flow.

\section{Methodology}

This section briefly describes the theoretical basis of the variance ellipse analysis from which the eddy anisotropy is derived; for a more complete review refer to Hoskins et al. (1983), Marshall et al. (2012), Waterman 83 Lilly (2015), and references therein.

The zonal $(u)$ and meridional $(v)$ velocities at a given location $\mathbf{x}(x, y, z)$ are Reynolds decomposed into their time-mean $(\bar{u}, \bar{v})$ and time-varying $\left(u^{\prime}\right.$, $\left.v^{\prime}\right)$ components:

$$
u(\mathbf{x}, t)=\bar{u}(\mathbf{x})+u^{\prime}(\mathbf{x}, t), \quad \text { and } \quad v(\mathbf{x}, t)=\bar{v}(\mathbf{x})+v^{\prime}(\mathbf{x}, t) .
$$

At location $\mathbf{x}$, the timeseries of the time-varying components of the horizontal velocity define a variance ellipse in $\left(u^{\prime}, v^{\prime}\right)$-space. The amplitude, eccentricity and orientation of this variance ellipse are defined in terms of the time-mean velocity variances $\overline{u^{\prime 2}}$ and $\overline{v^{\prime 2}}$ and covariance $\overline{u^{\prime} v^{\prime}}$. 
The characteristics of the variance ellipse can be derived explicitly from the velocity covariance matrix $\Sigma$ :

$$
\Sigma=\left[\begin{array}{ll}
\overline{u^{\prime 2}} & \overline{u^{\prime} v^{\prime}} \\
\overline{u^{\prime} v^{\prime}} & \overline{v^{\prime 2}}
\end{array}\right],
$$

which describes the time-mean horizontal eddy velocity variances and covariance. It is convenient to separate this eddy covariance matrix into its isotropic and anisotropic components:

$$
\Sigma=K \mathbf{I}+\mathbf{A},
$$

where $K$ is the eddy kinetic energy given by,

$$
K=\frac{\left(\overline{u^{\prime 2}}+\overline{v^{\prime 2}}\right)}{2},
$$

$\mathbf{I}$ is the identity matrix, and $\mathbf{A}$ is the anisotropic component of the eddy covariance matrix,

13

with

$$
\mathbf{A}=\left[\begin{array}{cc}
M & N \\
N & -M
\end{array}\right],
$$

$M$ and $N$ are composed of the eddy Reynolds stresses describing the eddy fluxes of horizontal momentum. These anisotropic elements of the eddy covariance matrix describe the tendency for the variability to align along a preferred axis and combine to give the portion of the eddy kinetic energy associated with anisotropic variability $L$ :

$$
L=\sqrt{M^{2}+N^{2}} .
$$

$L$ is subject to the bound that it must always be less than or equal to the total eddy kinetic energy, such that,

$$
\frac{L}{K} \leq 1
$$

The ratio $L / K$ is a measure of ellipse shape varying like the eccentricity between 0 and 1 and as such provides a useful measure of the degree of 
so-called "eddy anisotropy", the anisotropy associated with the eddy or fluctuating portion of the flow. As shown by Waterman \& Lilly (2015), the covariance matrix (Eqn. 3) can be written in terms of the total eddy kinetic energy, the anisotropic eddy kinetic energy and an orientation $\Theta$ as,

$$
\Sigma=K \mathbf{I}+\mathbf{A}=K \mathbf{I}+L\left[\begin{array}{cc}
\cos 2 \Theta & \sin 2 \Theta \\
\sin 2 \Theta & -\cos 2 \Theta
\end{array}\right]
$$

Here $\Theta$ is the orientation of the principle axis of anisotropy (Hoskins et al., 1983; see also Fig. 2 of Waterman $\&$ Hoskins, 2013), hereinafter referred to as the "eddy orientation". It is given by

$$
\Theta=\frac{1}{2} \tan ^{-1}\left(\frac{N}{M}\right)
$$

The orientation of eddy anisotropy relative to the orientation of the basic state ( mean) shear defines the direction of the energy conversion between the mean and eddy fields. For eddies to extract energy from the horizontal shear of the mean current, the anisotropy must be orientated such that the variance ellipses "lean" against the mean shear; for ellipses that tilt with the mean flow shear, eddies transfer energy from the eddy field to the basic current (Starr, 1968; see also Pedlosky, 1987 Section 7.3, and Fig. 4 of Marshall et al., 2012). It is clear from applying a coordinate rotation to Equation (9) that the first term $K \mathbf{I}$ is invariant to coordinate rotations, whilst the elements of the matrix comprising the second term will be modified by such a transformation; this indicates that $L$ is associated with anisotropy or directionality of the velocity covariance.

Here, the eddy anisotropy of surface flows in the global ocean is examined in satellite observations and a $1 / 12^{\circ}$ global ocean model. For the case of the model output, the vertical structure of anisotropy and its relationship with the underlying model bathymetry is also considered. In addition to the full flow, the eddy anisotropy of the ocean model is computed for the barotropic and baroclinic variability separately; this is achieved by first decomposing the horizontal velocities into their barotropic (depth average) and baroclinic (deviation from the depth average) flows at each spatial location, and then repeating the above calculations (Eqns. 1-10).

The practical computation of these terms is simplified through ensemble averaging, that is,

$$
\overline{u^{\prime 2}}=\overline{u^{2}}-\bar{u} \bar{u}, \quad \overline{v^{\prime 2}}=\overline{v^{2}}-\bar{v} \bar{v}, \quad \overline{u^{\prime} v^{\prime}}=\overline{u v}-\bar{u} \bar{v},
$$


where $\bar{u}, \bar{v}, \overline{u^{2}}, \overline{v^{2}}$ and $\overline{u v}$ are ideally accumulated online, or alternatively from stored velocity fields representative of the temporal variability; here the latter is employed. Obviously, it is important to ensure the zonal and meridional velocities are collocated, which is not always the case for ocean model output.

\section{Model and Observations}

This study uses output from the ORCA12 (see description in Treguier et al. , 2014) configuration of NEMO (Nucleus for European Modelling of the Ocean; Madec, 2008), run by the DRAKKAR Group (The DRAKKAR Group , 2007). ORCA12 simulates the global ice-ocean circulation with a latitudinally-dependent horizontal resolution that is largest at the equator $\left(9 \mathrm{~km}, 1 / 12^{\circ}\right)$ and decreases with the cosine of latitude towards the poles; the vertical resolution ranges from $5 \mathrm{~m}$ at the surface to $250 \mathrm{~m}$ at depth through 46 vertical levels. The model has been forced with the DRAKKAR Forcing Set \#4 (DFS4; The DRAKKAR Group, 2007; Brodeau et al. , 2010) to produce a hindcast simulation for the 10-year period of January 2003 to December 2012, with the velocity fields output and stored as 5-day averages. The timeseries of the zonal and meridional global velocity fields, and their barotropic and baroclinic components, are used to calculate the terms characterizing the eddy kinetic energy and its anisotropy $(K, L, \Theta)$.

In addition to the ORCA12 output, this study uses 10 years of geostrophic velocities derived from merged satellite altimetries; these altimetry products were produced by Ssalto/Duacs and distributed by AVISO, with support from CNES (http://www.aviso.oceanobs.com/duacs/). The velocities are provided as daily-averages on a regular $1 / 4^{\circ}$ grid and cover the same period as the model simulations (2003-2012). Considering the $\sim 10$ day repeat orbital cycle and objective remapping of along-track observations to the gridded product, the global $1 / 4^{\circ}$ altimetry record is expected to only be able to resolve a portion of the mesoscale variability, corresponding to eddies having an e-folding scale of greater than 30-45 km (see, for example, Chelton et al., 2011; Hallberg , 2013). Nevertheless it is useful to look at the eddy anisotropy in this data set globally as it contains valuable information about the surface geostrophic variability at the scales that are resolved (e.g., Ducet $\& 3 \mathrm{Le}$ Traon, 2001; Huang et al., 2007; Scott et al., 2008), and it offers guidance for understanding future estimates of eddy anisotropy derived from improved observational systems, such as the upcoming SWOT altimetry data (e.g., Du- 
rand et al., 2010; Fu et al., 2010). It is important to note that the different spatial and temporal resolutions of the model and altimetry data do not allow for a direct and fair comparison of calculated variabilities, and that the ambiguity of the altimetry data resolution (especially in regards to the scales of variability resolved) complicates the process of selectively-sampling the model output so as to provide a fair comparison for the altimetry product.

\section{Results}

This section presents the anisotropy of ocean variability calculated from the AVISO product and ORCA12 model output. The section is divided into four parts; the focus of the first is on the anisotropy of surface velocities, the second on the vertical structure of eddy anisotropy in the model velocity fields, the third on the eddy anisotropy of the barotropic and baroclinic velocities in the modelled Southern Ocean, and the fourth on the model eddy anisotropy in the vicinity of the Macquarie Ridge and Campbell Plateau. The focus on the Southern Ocean, and in particular the vicinity of Macquarie Ridge and Campbell Plateau, serve as illustrative examples of the distinction between eddy anisotropy of the barotropic and baroclinic velocity fields in eddy-rich regions where bathymetry is known to have a strong influence on the flow.

\subsection{Surface Eddy Anisotropy}

The AVISO product was used to characterize the global distribution of eddy anisotropy by calculating $K, L$ and their ratio (Fig. 1). The distribution of $L$ (Fig. 1(b)) shows many similarities with the distribution of $K$ (Fig. 1(a)), being largest in and surrounding regions of large flow speeds. However relative to $K$, the spatial distribution of $L$ appears more localized to the large flow speed regions and is also richer in mesoscale structure. The eddy anisotropy $L / K$ tends to be large where currents are strongly influenced by bathymetry or by equatorial dynamics, and exhibits complicated mesoscale structure (Fig. 1(c)). Whilst the distribution of $L / K$ is maximal along coastlines and shelfbreaks, and near the equator, the central basin regions tend to be more isotropic. The global average value of surface eddy anisotropy is 0.25 , implying that approximately $25 \%$ of surface eddy kinetic energy is associated with anisotropic variability (Tab. 1).

The eddy anisotropy of the ORCA12 model surface fields generally compares well with that derived from the satellite observations in both magnitude 
and spatial distributions, a consequence of the fact that both the eddy kinetic energy (Figs. 1(a) \& 2(a)) and its anisotropic component (Figs. 1(b) \& 2(b)) in the model and satellite observations are similar in magnitude and distribution. However, regions where the ORCA12 anisotropy is consistently greater than that of the AVISO-derived fields include the coastlines and equatorial latitudes, a consequence of the anisotropic eddy kinetic energy $L$ being significantly larger in the model fields (Figs. 1(b) \& 2(b)). Globally, the average eddy anisotropy in the model surface is 0.29 , implying that $29 \%$ of ORCA12 surface eddy kinetic energy is associated with anisotropic variability (Tab. 1); this is slightly more anisotropic than the AVISO-derived fields and is reflected in the global distributions of $L / K$ (Figs. 1 (c) \& 2(c)).

\subsection{Vertical Structure of Eddy Anisotropy}

The ORCA12 model output provides an opportunity to investigate the vertical structure of ocean variability and, in particular, eddy anisotropy. An initial step is to compare the anisotropy of surface variability with that of the bottom; for this, the horizontal velocities of the second-deepest nonbathymetry grid cell are used to calculate the bottom covariance fields, so as to avoid complications with viscous boundary conditions and bathymetry representation schemes. In contrast to the surface, the bottom variability is predominantly anisotropic, as reflected in the similarities between the bottom $K$ and bottom $L$ fields (Figs. 3(a) \& 3(b)). Although the global average of bottom eddy kinetic energy is almost 2 orders of magnitude smaller than the surface, the global average value of eddy anisotropy is 0.65 , implying that $65 \%$ of all bottom eddy kinetic energy is associated with directional or anisotropic variability (Tab. 1). The distribution of the bottom eddy anisotropy appears to be influenced by the underlying bathymetry; regions of significant bathymetric features (e.g., mid-ocean ridges, continental shelves, island chains) tend to be highly anisotropic, whilst the relatively featureless plains and basins (e.g., Aleutian, Admundsen, Argentine, Bellingshausen, Ceylon, Enderby, Weddell) are more isotropic (Fig. 3(c)).

Vertical sections of eddy anisotropy $L / K$ highlight this transition between the anisotropic bottom and relatively isotropic surface. The bottom intensification of eddy anisotropy is evident in the zonal average of $L / K$ (Fig. 4(a)), and with the exception of the equatorial regions, the eddy anisotropy generally increases monotonically with depth.

The anisotropic bottom signal tends to be localized to regions of sloping bathymetry whilst in regions of flat bathymetry, the bottom variability 
tends to be more isotropic. This is evident in the relative intensification of the bottom anisotropy when the calculation of the zonal average of $L / K$ is restricted to regions above sloping bathymetry (i.e. $\nabla H \neq 0$, where $H$ is the ocean model depth; Fig. 4(b)), compared to when it is restricted to regions of locally flat model bathymerty (i.e. $\nabla H=0$; Fig. 4(c)). This differing influence of flat or sloping bathymetry is readily observed in various basin transects of $L / K$, such as a circumpolar transect at $55^{\circ} \mathrm{S}$ (Fig. 5(a)). In regions of sloping bathymetry, the anisotropic bottom signal penetrates upwards $\sim 1000 \mathrm{~m}$ into the water column, occasionally reaching the ocean surface. In addition to the bottom intensification, the eddy anisotropy is notably enhanced downstream of major obstacles across the Antarctic Circumpolar Current (ACC), such as the Drake Passage, Kerguelen Plateau and Macquarie Ridge.

Vertical transects of the eddy orientation, $\Theta$, reveal that in spite of the significant vertical structure in eddy anisotropy, the eddy orientation remains relatively unchanged with depth (Fig. 5(b)). Note that while the cardinal direction of the eddy orientation is of little consequence (rather, the eddy orientation direction relative to that of the mean shear is important for diagnosing eddy-mean-flow interactions; see Waterman \& Lilly, 2015), the observation that the orientation of the eddy anisotropy maintains vertical coherence suggests a significant portion of the anisotropic signal is barotropic.

Given the apparent link between eddy anisotropy and sloping bathymetry, it is useful to consider the alignment of the eddy anisotropy relative to the direction of the underlying isobath (Fig. 6). This is an angle between 0 and $\pi / 2$, where 0 implies the eddy anisotropy is aligned with the isobath, and $\pi / 2$ implies they are perpendicular. At the surface, there is a slight tendency for the variability to align itself with the underlying isobath (Fig. $6(a))$; approximately $15 \%$ of the anisotropic surface eddy kinetic energy is aligned within $10^{\circ}$ of the underlying isobath. This effect is amplified at depth: bottom variability has an increased tendency to align with the local isobath (Fig. 6(b)); over 20\% of the anisotropic bottom eddy kinetic energy is aligned within $10^{\circ}$ of the underlying isobath.

\subsection{Barotropic vs. Baroclinic Eddy Anisotropy}

The barotropic tendency of eddy anisotropy warrants further investigation. Given the prevalence of Southern Ocean eddies, and their crucial role in the $\mathrm{ACC}$ and its vertical structure, the region south of $\sim 30^{\circ} \mathrm{S}$ serves 
as an illustrative example of the distinction between eddy anisotropy of the barotropic and baroclinic flows (e.g., Rintoul, 2010).

Separating the horizontal flow into its barotropic (depth-averaged) and baroclinic (deviation from the depth-average) components shows that over $60 \%$ of the total eddy kinetic energy in the ORCA12 Southern Ocean is contained in the barotropic flow (Tab. 2). The distribution of barotropic eddy anisotropy is highly variable and rich in mesoscale (10-100 km) structure; the barotropic variability is anisotropic on continental shelves, shelfbreaks, and plateaus, and its distribution resembles that of $|\nabla(f / H)|$ (where $f$ is the Coriolis parameter; compare Fig. 7(c) with $7(\mathrm{~g})$ ).

Baroclinic variability in the ORCA12 Southern Ocean contains less than $40 \%$ of the total eddy kinetic energy (Tab. 2). At the surface, the distribution of eddy anisotropy in the baroclinic flow is virtually unchanged from that of the full flow case (compare Fig. 7(b) with 7(d)), and the anisotropy is strongest where $|\nabla(f / H)|$ is large (Fig. $7(\mathrm{~g})$ ). The Southern Ocean average of baroclinic bottom $K$ is similar to the full flow case, whilst the baroclinic bottom $L$, and thus the eddy anisotropy $L / K$, almost halves (Tab. 2). This shift towards a more isotropic bottom baroclinic eddy field is most obvious in the distribution of baroclinic bottom $L / K$ (compare Fig. $7(\mathrm{e})$ with $7(\mathrm{f})$ ); for both the baroclinic and full flow cases, the bottom eddy anisotropy is weakest where $|\nabla(f / H)|$ is small (Fig. $7(\mathrm{~g}))$. Indeed, the vertical penetration of the strong bottom eddy anisotropy above sloping bathymetry that is evident in the full flow (Fig. 5(a)) is not present in the baroclinic flow (Fig. 8). Additionally, with the absence of the bottom intensification, the enhancement of eddy anisotropy downstream of major obstacles is more apparent.

A dominant feature of the circumpolar transect of baroclinic $L / K$ is an intermediate-depth maximum in eddy anisotropy. This is an artifact of the baroclinic decomposition; at depths where the current velocity in a given direction is equal to the barotropic velocity (for example, $u=u_{B T}$ or $v=$ $v_{B T}$ ), the baroclinic velocity becomes zero, leading to an anisotropizing of the variability in the perpendicular direction. This feature also suggests that for ORCA12 the majority of the baroclinic variability at this latitude is contained in the first baroclinic mode, such that $u=u_{B T}$ or $v=v_{B T}$ at only one depth in the water column.

\subsection{Eddy Anisotropy at the Macquarie Ridge and Campbell Plateau}

Given the rich spatial structure of eddy anisotropy, and its apparent relationship with bathymetry, it is useful to consider the eddy anisotropy in a 
localized region with significant bathymetry that is known to influence the flow. One such region is the Macquarie Ridge; this ridge presents a large bathymetric obstacle for the ACC, which crosses the ridge primarily through a few narrow gaps. A major gap in the ridge is north of Macquarie Island at $\left(53.3^{\circ} \mathrm{S}, 159.4^{\circ} \mathrm{E}\right)$ where a flow equivalent to almost one-third of the total ACC transport crosses the ridge as a predominantly barotropic and steady current; it is one of the rare places in the ocean where the mean kinetic energy is observed to exceed the eddy kinetic energy (Rintoul et al. , 2014). Eastward of the Macquarie Ridge, the ACC encounters Campbell Plateau where, rather than flowing over the Plateau, the ACC tends to flow along the slope as an energetic and highly variable current (Stanton \& Morris, 2004).

In the model, the mean surface flow is fastest across the Macquarie Ridge at the $\left(53.3^{\circ} \mathrm{S}, 159.4^{\circ} \mathrm{E}\right)$ gap, and remains at current speeds of $>0.2 \mathrm{~m} \mathrm{~s}^{-1}$ as it flows along the southeastern edge of Campbell Plateau (Fig. 9(a)). This mean flow pattern is largely maintained with depth; the flow through the gap and that rounding Campbell Plateau are connected by the $>0.1 \mathrm{~m} \mathrm{~s}^{-1}$ contour to a depth of $\sim 2000 \mathrm{~m}$ (Figs. 9(b)-(d)). The surface variability in the region is mostly isotropic except in shallow waters, against coastlines, and above the eastern and western flanks of the Macquarie Island ridge section and the steeper edges of Campbell Plateau (Fig. 9(a)). The variability becomes more anisotropic with depth, especially against the sloping bathymetry (Figs. 9(b)-(d)). In agreement with observations (e.g., Rintoul et al., 2014), the flow through the $\left(53.3^{\circ} \mathrm{S}, 159.4^{\circ} \mathrm{E}\right)$ gap is predominantly barotropic with the current speed remaining over $0.1 \mathrm{~m} \mathrm{~s}^{-1}$ beyond $3000 \mathrm{~m}$ depth. The eddy kinetic energy $K$ (not shown here) is a local minimum in the flow through the gap. Any variability within the gap is isotropic at all depths except against the ridge flanks. Immediately upstream to the north of the gap, the variability is highly anisotropic below depths of $1000 \mathrm{~m}$, indicative of eddymean-flow interaction at depth (Fig. 9(c)-(d)). After crossing the Macquarie Ridge, the current flows along the southeastern edge of the Campbell Plateau where the bathymetry is steepest. The variability is highly anisotropic along the Plateau edge at all depths including the surface; this anisotropic feature is evident in the satellite observations (Fig. 7(a)) and previous studies (e.g., Stanton \& Morris , 2004), and is presumably related to the presence of the bathymetric slope. 


\section{Discussion}

There is good agreement between the satellite and model surface distributions of $K, L$, and $L / K$. Both exhibit large values of $K$ in and surrounding the regions of large flow speed, especially in the western boundary currents, equatorial currents and the Antarctic Circumpolar Current (ACC). The distributions of the anisotropic eddy kinetic energy $L$ exhibit more mesoscale structure than their respective distributions of $K ; L$ appears more localized and confined to the regions of large flow speeds. The distributions of $L / K$ reveal that the largest surface eddy anisotropy occurs along the coastlines and in the vicinity of the equator. The strong eddy anisotropy along the coastlines can be understood by considering the constraint the coastline imposes on the onshore flow and its fluctuations: the onshore flow and fluctuations are smaller than the alongshore flow and fluctuations. At and near the equator, the eddy orientation (not shown here) suggests that the strong eddy anisotropy is due to a combination of equatorial Kelvin waves elongating fluctuations zonally and tropical instability waves elongating fluctuations meridionally.

The surface variability fields derived from the satellite altimetry are generally more isotropic than those of the model. This is likely due to the reduced spatial resolution of the satellite observations, and perhaps in part to the objective remapping procedure used to generate a daily $1 / 4^{\circ}$ global product from along-track altimetry measurements. Irrespective of the cause of this apparent isotropic bias relative to the model, it does suggest that any anisotropic signals detected by the satellite observations are indeed robust features of the circulation. Therefore, the common anisotropic signals found in the AVISO and model surface fields (such as enhanced anisotropy on the Campbell and Kerguelen Plateaus, Macquarie Ridge, Drake Passage, etc.) are likely to result from common causes, and as such we expect that the model provides the means to diagnose such causes.

Ocean variability tends to be anisotropic in the vicinity of major bathymetric features, such as coastlines, shelfbreaks, seamounts or plateaus, and this relationship between eddy anisotropy and $f / H$ is worthy of further discussion. Previous studies have drawn attention to the effect of $f / H$ on shaping the eddy anisotropy (e.g., Morrow et al., 1994; Stanton 85 Morris , 2004; Scott et al. , 2008), and generally conclude that, with the exception of a few specific regions, the alignment of surface variability is not sensitive to the direction of underlying isobaths. This is consistent with the analysis of 
surface variability presented here, especially in regions of weakly barotropic flows (compare Figs. 7(b) with 7(g)). However, the alignment of sub-surface variability is found to be strongly influenced by bathymetry; this link between anisotropic eddy kinetic energy and bathymetry is most apparent in bottom fluctuations. Although the bottom eddy kinetic energy is about two orders of magnitude smaller than that at the surface, where the bathymetry is sloping the bottom variability is almost entirely anisotropic and aligned with the underlying isobaths. Interestingly, the regions of largest bottom $K$ coincide with regions of isotropic variability and flat bathymetry unable to support topographic wave activity, indicative of $f$-plane turbulence. These findings are consistent with known dynamics of variability near bathymetry, such as bottom-trapped Rossby waves (Rhines , 1970) and the topostrophic processes encompassed in the Neptune Effect (Holloway, 2008), and can offer guidance for shaping parameterizations for the unresolved variability based on the underlying bathymetric slope. Such a parameterization scheme for bottom variability is especially warranted considering the current ocean modelling practice for $z$-coordinate models to reduce vertical resolution with depth.

Further insight into the vertical structure of ocean variability is gained by partitioning the flow into its barotropic and baroclinic components. The isobath-aligned anisotropic bottom variability is contained in the barotropic flow. This makes good physical sense; given that the barotropic flow tends to follow contours of constant $f / H$, it should come as no surprise that the barotropic variability follows suit (compare Figs. $7(\mathrm{c})$ with $7(\mathrm{~g})$ ). On the other hand, the baroclinic flow is less constrained by $f / H$ contours, and this is reflected in the finding that bathymetric slope appears to have little influence on the baroclinic variability (compare Figs. $7(\mathrm{~d})$ with $7(\mathrm{~g})$ ).

The fundamental difference in the nature of the barotropic and baroclinic variability suggests there may be benefit in distinct parameterizations for unresolved barotropic and unresolved baroclinic variability. One approach could take advantage of the fact that, given the separation of time- and length-scales between barotropic and baroclinic dynamics, many ocean models solve these flows separately with a fast two-dimensional barotropic solver sub-cycled within the slower three-dimensional baroclinic solution (e.g., Killworth et al., 1991; Griffies et al., 2001). This separation presents an opportunity to parameterize the unresolved barotropic variability independent of the baroclinic variability and in a way that reflects its tendency to be anisotropic and aligned with the underlying isobath. Presumably, a parameterization for 
unresolved barotropic variability would be more active in models that overpartition energy to the barotropic and low baroclinic modes, and be relatively idle in ocean models with greater vertical resolution.

Efforts to capture the effect of eddies in ocean models have focused on improving the representation of lateral eddy structures through increased horizontal resolution (e.g., Hallberg $\mathscr{G}$ Gnanadesikan, 2006), whilst relatively less attention has gone to improving the representation of the vertical structure of the eddy field. The vertical structure of ocean variability presented here highlights the need for increases of the vertical resolution to track with those of the horizontal resolution. Indeed, atmospheric studies have identified substantial benefits to increasing both the horizontal and vertical resolution consistently (Roeckner et al., 2006). It may be the case that ocean models with $1 / 12^{\circ}$ horizontal resolution require $>46$ vertical levels to resolve the dynamics of the finer lateral structures. For instance, it is obvious that there is little physical benefit to laterally resolving the high-order baroclinic Rossby deformation radii when the vertical resolution only permits low-order baroclinic and barotropic mode dynamics, and vice-versa.

The present study has focused on the horizontal eddy anisotropy to highlight the structure and distribution of the eddy anisotropy globally and as a function of depth. A topic of ongoing work is to incorporate eddy buoyancy flux terms into the analysis. To do this from the perspective of eddy geometry involves the characterization of a 3D variance ellipsoid in momentumbuoyancy eddy flux space in place of the 2D variance ellipse employed here. It is expected that these eddy buoyancy flux terms will also have distinctive vertical structure, and interact with the eddy momentum field primarily through the baroclinic eddies. Additional utility of using the eddy geometry framework to characterize and understand eddy fluxes potentially lies in understanding the time dependence of the eddy geometry, such as seasonality, or in the case of barotropic variability, diurnal/tidal cycles.

\section{Conclusion}

Quantifying the anisotropy of eddy variability is important as eddy feedbacks on the larger scale flow result solely from the anisotropic components of the eddy variability. Here, the anisotropy of ocean variability has been examined in satellite observations and a $1 / 12^{\circ}$ global ocean model. It is found that the anisotropy of eddy variability is largest near the equator, along coastlines and close to bathymetry. Eddy anisotropy is found to have a dis- 
Table 1: Global averages of $K\left(\mathrm{~m}^{2} \mathrm{~s}^{-2}\right), L\left(\mathrm{~m}^{2} \mathrm{~s}^{-2}\right)$, and $L / K$ for surface geostrophic velocities derived from the AVISO product and for the surface, bottom and depth-averaged ORCA12 model output.

\begin{tabular}{|c||c||c|c|c|}
\hline \multirow{2}{*}{ Term } & \multirow{2}{*}{} & \multicolumn{3}{c|}{ ORCA12 } \\
\cline { 3 - 5 } & AVISO & Surface & Bottom & All \\
\hline$K$ & $2.34 \mathrm{e}-2$ & $2.42 \mathrm{e}-2$ & $5.57 \mathrm{e}-4$ & $2.17 \mathrm{e}-3$ \\
$L$ & $6.37 \mathrm{e}-3$ & $8.81 \mathrm{e}-3$ & $3.12 \mathrm{e}-4$ & $6.90 \mathrm{e}-4$ \\
$L / K$ & 0.245 & 0.294 & 0.644 & 0.418 \\
\hline
\end{tabular}

tinctive vertical structure and become increasingly anisotropic with depth, especially in the vicinity of sloping bathymetry. The highly anisotropic bottom variability tends to be aligned with the underlying isobaths, which is useful knowledge given that eddy-mean-flow interactions are sensitive to the orientation of the eddy anisotropy, and in particular the extent to which the eddy anisotropy orientation departs from the direction of the mean-flow shear. Partitioning the eddy variability into the barotropic and baroclinic components reveals that the anisotropic bottom variability is predominantly barotropic, whilst the upper-ocean variability is relatively baroclinic. Given the distinct natures of barotropic and baroclinic variability, the findings suggest that parameterizations for unresolved variability in ocean models may be improved by the development of a parameterization for unresolved barotropic Reynolds stresses that is based on the shape of the underlying bathymetry and operates only on the barotropic flow.

\section{Acknowledgments}

The altimeter products were produced by Ssalto/Duacs and distributed by AVISO, with support from CNES (http://www.aviso.oceanobs.com/duacs/). Many thanks to A. Hogg, B. Fox-Kemper and D. Marshall for very helpful discussions, and to the DRAKKAR Group for making available the ORCA12 model output. The numerical simulation was performed on CINES supercomputer (Montpellier, France). The work of KDS \& SW was supported by Australian Research Council grant RM10240. The work of PS was supported by the Australian Research Council grant DE150100223. The work of JML was supported by United States National Science Foundation grant \#OCE-1235310. MHE was supported by Australian Research Council grants FL100100214 \& CE110001028. 
Table 2: Southern Ocean (south of $30^{\circ} \mathrm{S}$ ) averages of $K\left(\mathrm{~m}^{2} \mathrm{~s}^{-2}\right), L\left(\mathrm{~m}^{2} \mathrm{~s}^{-2}\right)$, and $L / K$ for the surface, bottom and depth-averaged ORCA12 model output. These terms are calculated for the Southern Ocean using (i) the full flow, (ii) only the barotropic flow, and (iii) only the baroclinic flow of the ORCA12 model output.

\begin{tabular}{|c|c|c|c|c|c|c|c|c|}
\hline \multirow[b]{2}{*}{ Term } & \multirow[b]{2}{*}{ AVISO } & \multicolumn{3}{|c|}{ ORCA12 Southern Ocean } & \multirow{2}{*}{$\begin{array}{c}\text { ORCA12 } \\
\text { Barotropic }\end{array}$} & \multicolumn{3}{|c|}{ ORCA12 Baroclinic } \\
\hline & & Surface & Bottom & All & & Surface & Bottom & All \\
\hline$K$ & $1.53 \mathrm{e}-2$ & $1.61 \mathrm{e}-2$ & $8.14 \mathrm{e}-4$ & $2.82 \mathrm{e}-3$ & $1.72 \mathrm{e}-3$ & $9.01 \mathrm{e}-3$ & $8.40 \mathrm{e}-4$ & $1.09 \mathrm{e}-3$ \\
\hline$L$ & $3.13 \mathrm{e}-3$ & $3.46 \mathrm{e}-3$ & $3.65 \mathrm{e}-4$ & $6.86 \mathrm{e}-4$ & $4.28 \mathrm{e}-4$ & $1.84 \mathrm{e}-3$ & $2.27 \mathrm{e}-4$ & $2.41 \mathrm{e}-4$ \\
\hline$L / K$ & 0.218 & 0.222 & 0.588 & 0.361 & 0.277 & 0.205 & 0.314 & 0.262 \\
\hline
\end{tabular}

Figure 1: Distributions of (a) $K\left(\mathrm{~m}^{2} \mathrm{~s}^{-2}\right)$, (b) $L\left(\mathrm{~m}^{2} \mathrm{~s}^{-2}\right)$, and (c) $L / K$ derived from the $1 / 4^{\circ}$ AVISO product of surface geostrophic velocities for the period of 2003-2012. The $0.22 \mathrm{~m} \mathrm{~s}^{-1}$ time-mean speed contour is shown in grey.

Figure 2: Surface distributions of (a) $K\left(\mathrm{~m}^{2} \mathrm{~s}^{-2}\right)$, (b) $L\left(\mathrm{~m}^{2} \mathrm{~s}^{-2}\right)$, and (c) $L / K$ derived from the surface velocities of the $1 / 12^{\circ}$ ORCA12 ocean model for the period of 2003-2012. The $0.22 \mathrm{~m} \mathrm{~s}^{-1}$ time-mean speed contour is shown in grey.

Figure 3: Bottom distributions of (a) $K\left(\mathrm{~m}^{2} \mathrm{~s}^{-2}\right)$, (b) $L\left(\mathrm{~m}^{2} \mathrm{~s}^{-2}\right)$, and (c) $L / K$ derived from the horizontal velocities of the second-deepest grid cell of the $1 / 12^{\circ}$ ORCA12 ocean model for the period of 2003-2012. The $0.1 \mathrm{~m} \mathrm{~s}^{-1}$ time-mean speed contour is shown in grey.

Figure 4: Zonal averages of $L / K$ south of $\sim 30^{\circ} \mathrm{N}$ derived from the ORCA12 horizontal velocities for (a) all regions, (b) regions over sloping model bathymetry $(\nabla H \neq 0$, where $H$ is the local model ocean depth), and (c) regions over flat model bathymetry $(\nabla H=0)$. The $0.02 \mathrm{~m} \mathrm{~s}^{-1}$ zonal average of the time-mean zonal velocity is contoured (solid eastward, dashed westward). 
Figure 5: Circumpolar transects of (a) $L / K$ and (b) $\Theta$ derived from the ORCA12 horizontal velocities at $55^{\circ} \mathrm{S}$. The $0.1 \mathrm{~m} \mathrm{~s}^{-1}$ time-mean speed contour is shown in black. Locally flat model bathymetry $(\nabla H=0)$ is indicated in white. Note that for $\Theta$, north (red) is equivalent to south (blue), however for zonal mean-flow it is useful to identify regions where the eddy anisotropy orientation transitions through the north-south direction, hence the non-periodic colorbar.

Figure 6: Histograms of the angle between $\Theta$ and the local isobath for the (a) surface and (b) bottom of ORCA12 anisotropic variability in regions of sloping model bathymetry $(\nabla H \neq 0)$. The dashed lines represent uniform distributions (i.e. what would be expected if there were no connection between $\Theta$ and the local isobath). The local maxima in bottom eddy orientation at $\pi / 4$ is related to Ekman boundary layer dynamics; the second-lowest grid cell is occasionally entirely contained within the lower Ekman layer.

Figure 7: Surface distributions of Southern Ocean $L / K$ derived from (a) AVISO surface geostrophic velocities, (b) ORCA12 surface layer velocities, (c) the ORCA12 barotropic velocities, and (d) the ORCA12 baroclinic velocities; the $0.22 \mathrm{~m} \mathrm{~s}^{-1}$ time-mean speed contour is shown in grey. The distributions of Southern Ocean $L / K$ derived from (e) bottom ORCA12 velocities, and (f) the bottom ORCA12 baroclinic velocities; the 0.1 $\mathrm{m} \mathrm{s}^{-1}$ time-mean speed contour is shown in grey. The $(\mathrm{g})$ distribution of ORCA12 Southern Ocean $|\nabla(f / H)|\left(\mathrm{m}^{-2} \mathrm{~s}^{-1}\right)$.

Figure 8: Circumpolar transect of $L / K$ at $55^{\circ} \mathrm{S}$ derived from the baroclinic velocities of ORCA12. The $0.1 \mathrm{~m} \mathrm{~s}^{-1}$ time-mean speed contour is shown in black. Locally flat model bathymetry $(\nabla H=0)$ is indicated in white. The intermediate-depth maxima in $L / K$ relates to the depth where the current speed is equal to the barotropic speed, so that the baroclinic flow is zero.

Figure 9: Distributions of $L / K$ in the vicinity of Macquarie Ridge and Campbell Plateau at (a) the surface, (b) $1000 \mathrm{~m}$, (c) $2000 \mathrm{~m}$ and (d) $3000 \mathrm{~m}$ depth. The 1000, 2000 and $3000 \mathrm{~m}$ model isobaths are contoured in magenta. The $0.2 \mathrm{~m} \mathrm{~s}^{-1}$ time-mean speed contour is contoured in black for the surface (a); the $0.1 \mathrm{~m} \mathrm{~s}^{-1}$ time-mean speed contour is contoured for other depths $(b-d)$. 


\section{Bibliography}

Brodeau, L., B. Barnier, A.-M. Treguier, T. Penduff, and S. Gulev (2010), An ERA40-based atmospheric forcing for global ocean circulation models. Ocean Modelling, 31, 88-104. DOI: 10.1016/J.OCEMOD.2009.10.005.

Chelton, D. B., M. G. Schlax, and R. M. Samelson (2011), Global observations of nonlinear mesoscale eddies. Progress in Oceanography, 91, 167216. DOI: 10.1016/J.POCEAN.2011.01.002.

Ducet, N., P.-Y. Le Traon, and G. Reverdin (2000), Global highresolution mapping of ocean circulation from TOPEX/Poseidon and ERS1 and -2. Journal of Geophysical Research, 105, 19477-19498. DOI: 10.1029/2000JC900063.

Ducet, N., and P.-Y. Le Traon (2001), A comparison of surface eddy kinetic energy and Reynolds stresses in the Gulf Stream and the Kuroshio Current Systems from merged TOPEX/Poseidon and ERS-1/2 altimetric data. Journal of Geophysical Research, 106, 16603-16622. DOI: 10.1029/2000JC000205.

Dufour, C. O., J. Le Sommer, M. Gehlen, J. C. Orr, J.-M. Molines, J. Simeon, and B. Barnier (2013), Eddy compensation and controls of the enhanced sea-to-air $\mathrm{CO}_{2}$ flux during positive phases of the Southern Annular Mode. Global Biogeochemical Cycles, 27, 950-961. DOI: 10.1002/GBC.20090.

Durand, M., L.-L. Fu, D. P. Lettenmaier, D. E. Alsdorf, E. Rodrigues, and D. Esteban-Fernandez (2010), The Surface Water and Ocean Topography mission: Observing terrestrial surface water and oceanic submesoscale eddies. Proceedings of IEEE, 98, 766-779. DOI: 10.1109/JPROC.2010.2043031.

Eden, C. (2010), Parameterising meso-scale eddy momentum fluxes based on potential vorticity mixing and a gauge term. Ocean Modelling, 32, 58-71. DOI: 10.1016/J.OCEMOD.2009.10.008.

Fox-Kemper, B., R. Lumpkin, and F. O. Bryan (2013), Lateral transport in the ocean interior. Ocean Circulation and Climate: A 21st Century Perspective, International Geophysical Series, 103, Elsevier, 185-210. DOI: 10.1016/B978-0-12-391851-2.00008-8. 
Fu, L.-L., D. B. Chelton, P.-Y. Le Traon, and R. Morrow (2010), Eddy dynamics from satellite altimetry. Oceanography, 23, 14-25. DOI: 10.5670/OCEANOG.2010.02.

Gent, P. R. and J. C. McWilliams (1990), Isopycnal mixing in ocean circulation models. Journal of Physical Oceanography, 20, 150-155.

Gent, P. R. and J. C. McWilliams (1996), Eliassen-Palm Fluxes and the momentum equation in non-eddy-resolving ocean circulation models. Journal of Physical Oceanography, 26, 2539-2546.

Griffies, S. M., R. Pacanowski, M. Schmidt, and V. Balaji (2001), Tracer conservation with an explicit free surface method for z-coordinate ocean models. Monthly Weather Review, 129, 1081-1098.

Hallberg, R. and A. Gnanadesikan (2006), The role of eddies in determining the structure and response of the wind-driven southern hemisphere overturning: results from the Modelling Eddies in the Southern Ocean (MESO) Project. Journal of Physical Oceanography, 36, 2232-2252. DOI: 10.1175/JPO2980.1.

Hallberg, R. (2013), Using a resolution function to regulate parameterizations of oceanic mesoscale eddy effects. Ocean Modelling, 72, 92-103. DOI: 10.1016/J.OCEMOD.2013.08.007.

Holloway, G. (2008), Observing global ocean topostrophy. Journal of Geophysical Research, 113, C07054. DOI: 10.1029/2007JC004635.

Hoskins, B. J., I. N. James, and G. H. White (1983), The shape, propagation and mean-flow interaction of large-scale weather systems. Journal of the Atmospheric Sciences, 40, 1595-1612.

Huang, H.-P., A. Kaplan, E. N. Curchitser, and N. A. Maximenko (2007), The degree of anisotropy for mid-ocean currents from satellite observations and an eddy-permitting model simulation. Journal of Geophysical Research, 112, C09005, DOI: 10.1029/2007JC004105.

Killworth, P. D., D. Stainforth, D. J. Webb, and S. M. Paterson (1991), The development of a free-surface Bryan-Cox-Semtner ocean model. Journal of Physical Oceanography, 21, 1333-1348. 
Madec, G. (2008), NEMO Ocean Engine. Note du Pôle de Modélisation de l'Institut Pierre-Simon Laplace, 27.

Marshall, J. C. (1984), Eddy-mean-flow interaction in a barotropic ocean model. Quarterly Journal of the Royal Meteorological Society, 110, 573590.

Marshall, D. P., J. R. Maddison, and P. S. Berloff (2012), A framework for parameterizing eddy potential vorticity fluxes. Journal of Physical Oceanography, 42, 539-557. DOI: 10.1175/JPO-D-11-048.1.

McWilliams, J. C. (2008), The nature and consequences of oceanic eddies. Geophysical Monograph Series, 177, 5-15. DOI: 10.1029/177GM03.

Morrow, R., R. Coleman, J. Church, and D. Chelton (1994), Surface eddy momentum flux and velocity variances in the Southern Ocean from Geosat altimetry. Journal of Physical Oceanography, 24, 2050-2071.

Pedlosky, J. (1987), Geophysical Fluid Dynamics, Second Edition. SpringerVerlag.

Plumb, R. A. (1986), Three-dimensional propagation of transient quasigeostrophic eddies and its relationship with the eddy forcing of the timemean flow. Journal of the Atmospheric Sciences, 43, 1657-1678.

Preisendorfer, R. W. (1988), Principal component analysis in meteorology and oceanography. Elsevier, 425 pp.

Rhines, P. (1970), Edge-, bottom-, and Rossby waves in a rotating stratified fluid. Geophysical Fluid Dynamics, 1, 273-302.

Rintoul, S. R. (2010), Antarctic Circumpolar Current. Ocean Currents: A Derivative of the Encyclopedia of Ocean Sciences, 196.

Rintoul, S. R., S. Sokolov, M. J. M. Williams, B. Peña Molino, M. Rosenberg, and N. L. Bindoff (2014), Antarctic Circumpolar Current transport and barotropic transition at Macquarie Ridge. Geophysical Research Letters, 41, 7254-7261. DOI: 10.1002/2014GL061880.

Roeckner, E., R. Brokopf, M. Esch, M. Giorgetta, S. Hagemann, L. Kornblueh, E. Manzini, U. Schlese, and U. Schulzweida (2006), Sensitivity of simulated climate to horizontal and vertical resolution in the 
ECHAM5 atmosphere model. Journal of Climate, 19, 3771-3791. DOI: 10.1175/JCLI3824.1.

Scott, R. B., B. K. Arbic, C. L. Holland, A. Sen, and B. Qiu (2008), Zonal versus meridional velocity variance in satellite observations and realistic and idealized ocean circulation models. Ocean Modelling, 23, 102-112. DOI: 10.1016/J.OCEMOD.2008.04.009.

Stanton, B. R. and M. Y. Morris (2004), Direct velocity measurements in the Subantarctic Front and over Campbell Plateau, southeast of New Zealand. Journal of Goephysical Research, 109, C01028. DOI: 10.1029/2002JC001339.

Starr, V. P. (1968), Physics of Negative Viscosity Phenomena. McGraw-Hill, New York, 256pp.

The DRAKKAR Group (2007), Eddy-permitting ocean circulation hindcasts of past decades. CLIVAR Exchanges, 12, 3.

Treguier, A.-M., J. Deshayes, J. Le Sommer, C. Lique, G. Madec, T. Penduff, J.-M. Molines, B. Barnier, R. Bourdalle-Badie, and C. Talandier (2014), Meridional transport of salt in the global ocean from an eddy-resolving model. Ocean Science, 10. DOI: 10.5194/OS-10-243-2014.

Waterman, S., N. G. Hogg, and S. R. Jayne (2011), Eddy-mean flow interactions in the Kuroshio Extension region. Journal of Physical Oceanography, 41, 1182-1208. DOI: 10.1175/2010JPO4564.1.

Waterman, S. and B. J. Hoskins (2013), Eddy shape, orientation, propagation, and mean flow feedback in western boundary current jets. Journal of Physical Oceanography, 43, 1666-1690. DOI: 10.1175/JPO-D-12-0152.1.

Waterman, S. and J. M. Lilly (2015), A geometric decomposition of eddy feedbacks in barotropic systems. Journal of Physical Oceanography, 45, 1009-1024. DOI: 10.1175/JPO-D-14-0177.1.

Wunsch, C. (2007), The past and future ocean circulation from a contemporary perspective. Ocean Circulation: Mechanisms and Impacts, Geophysical Monograph Series, 173, American Geophysical Union, 53-74. DOI: 10.1029/173GM06. 


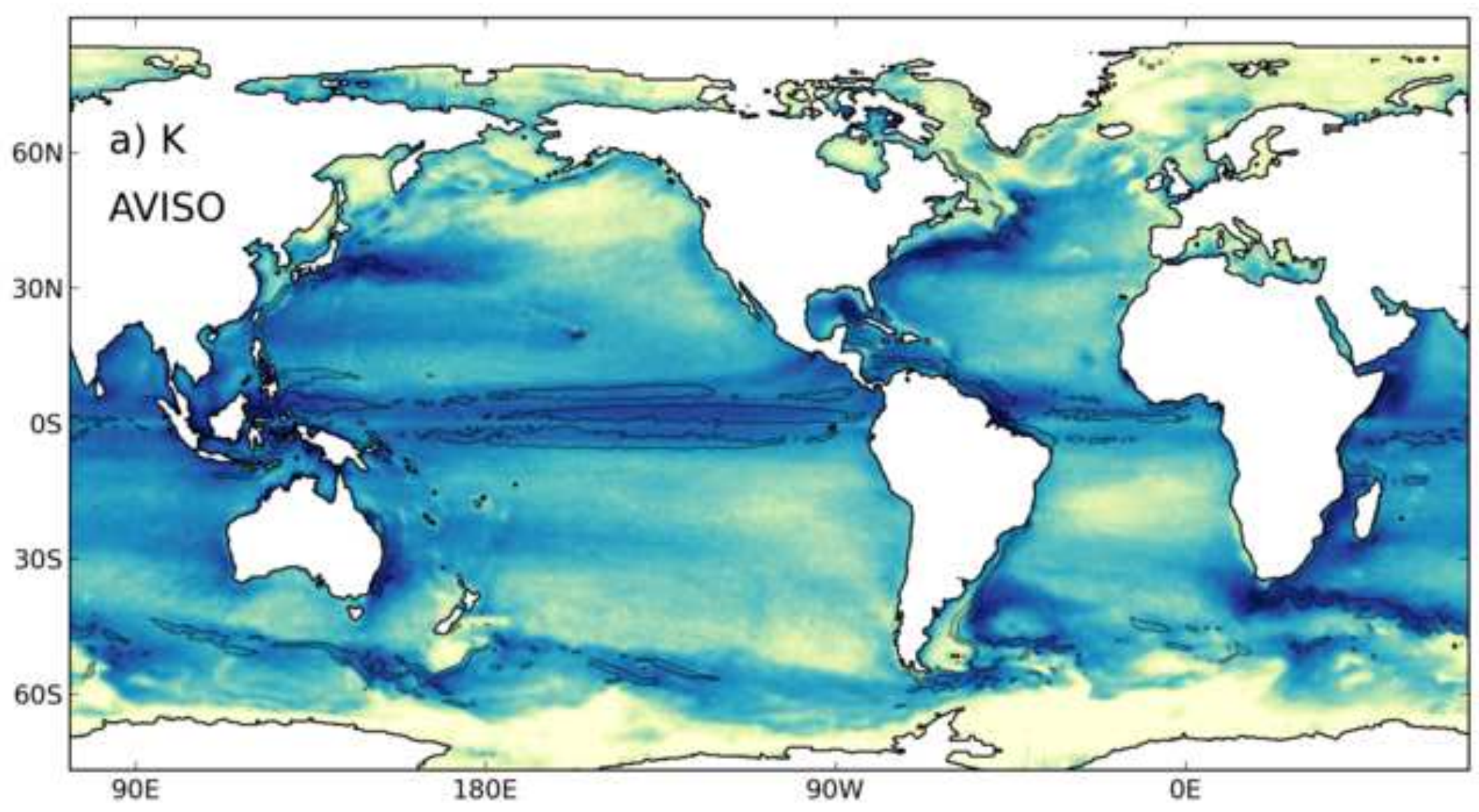

1e-1

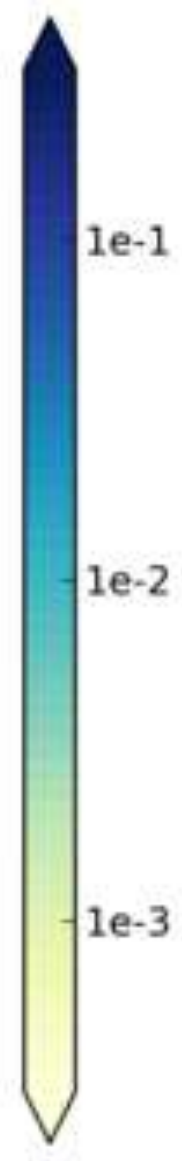




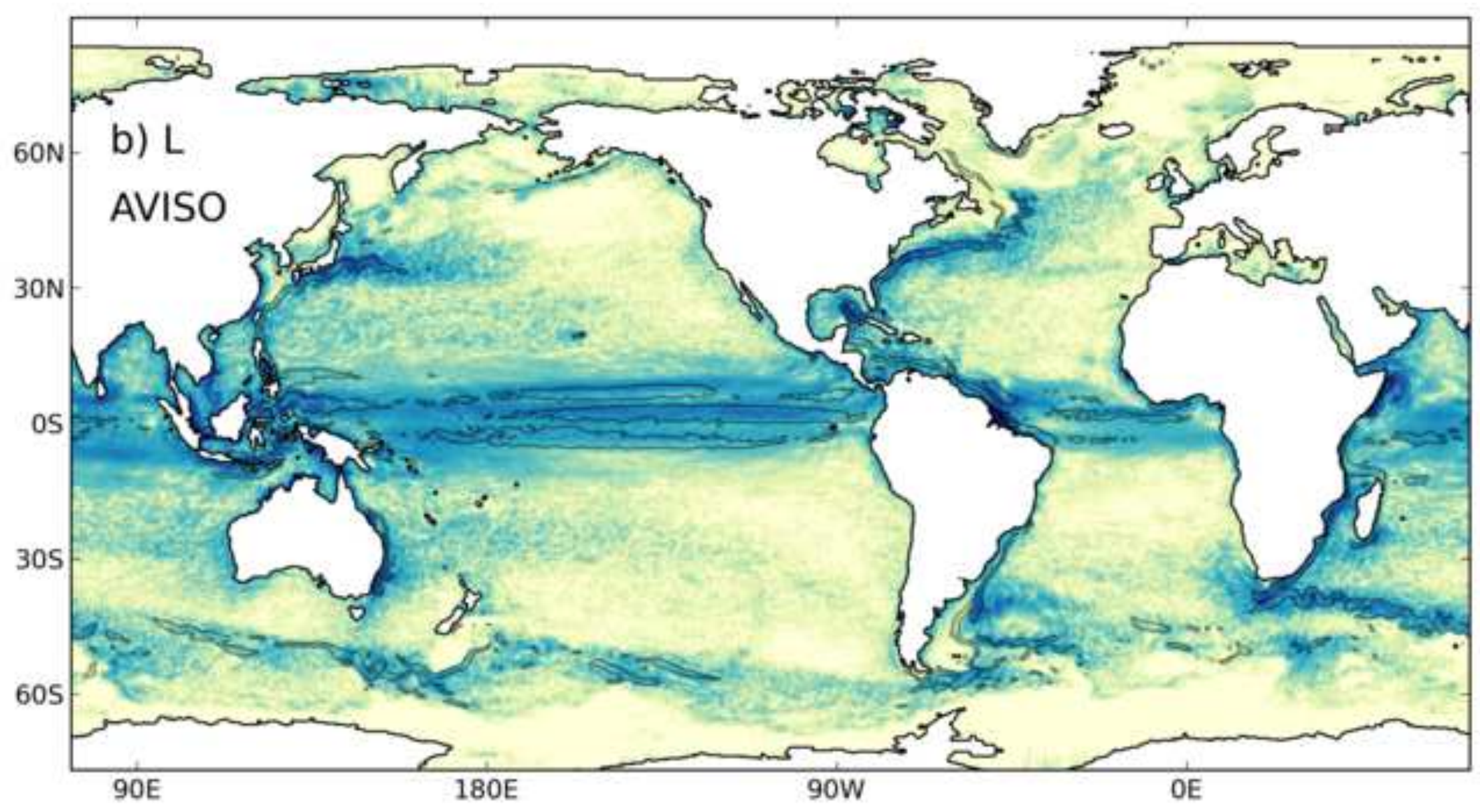

1e-1

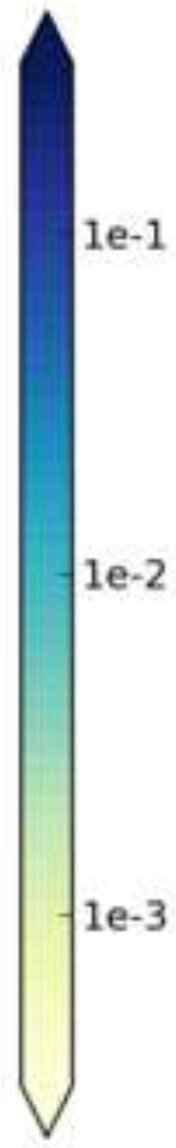



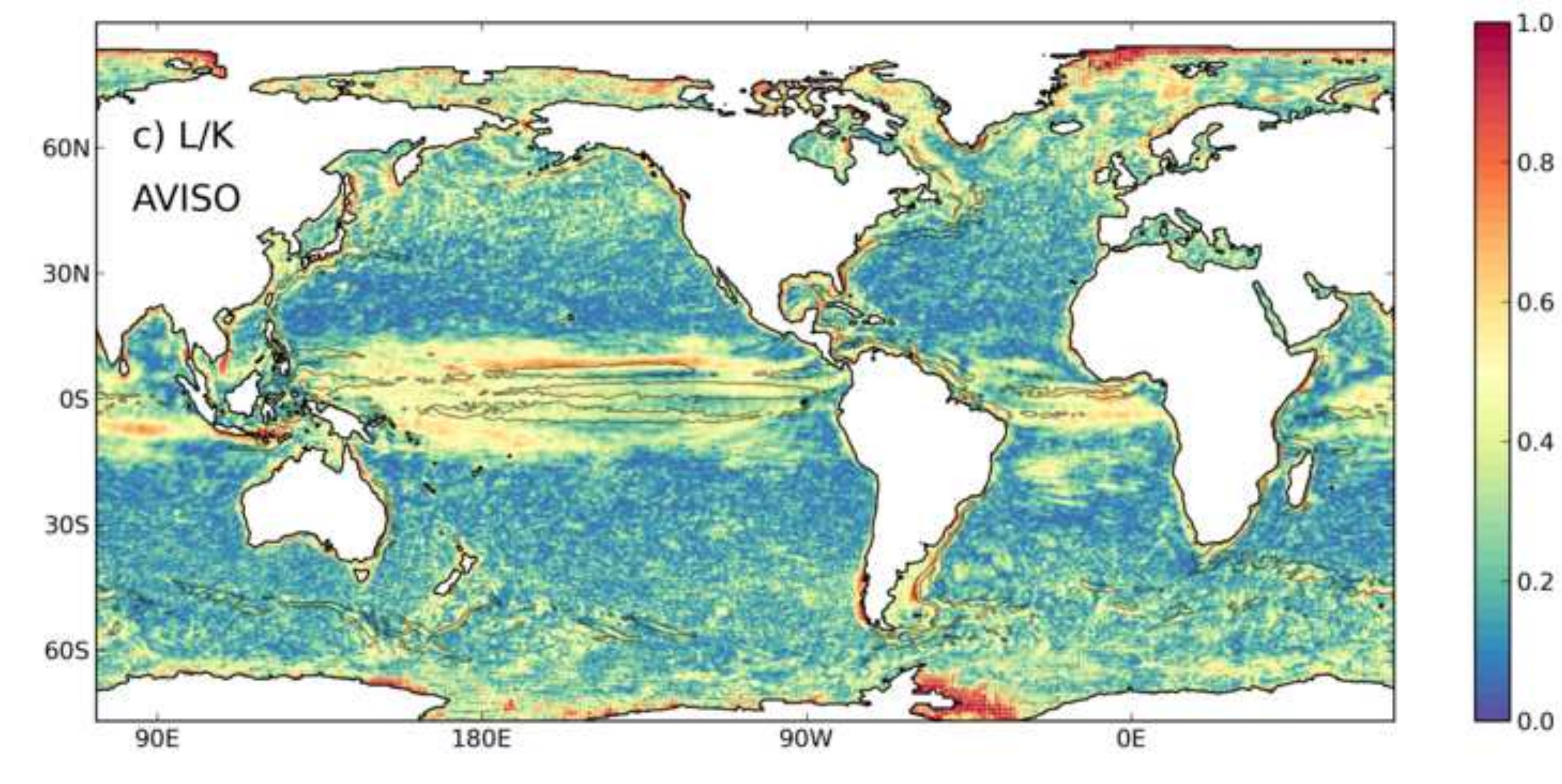

$$
90 \mathrm{E}
$$



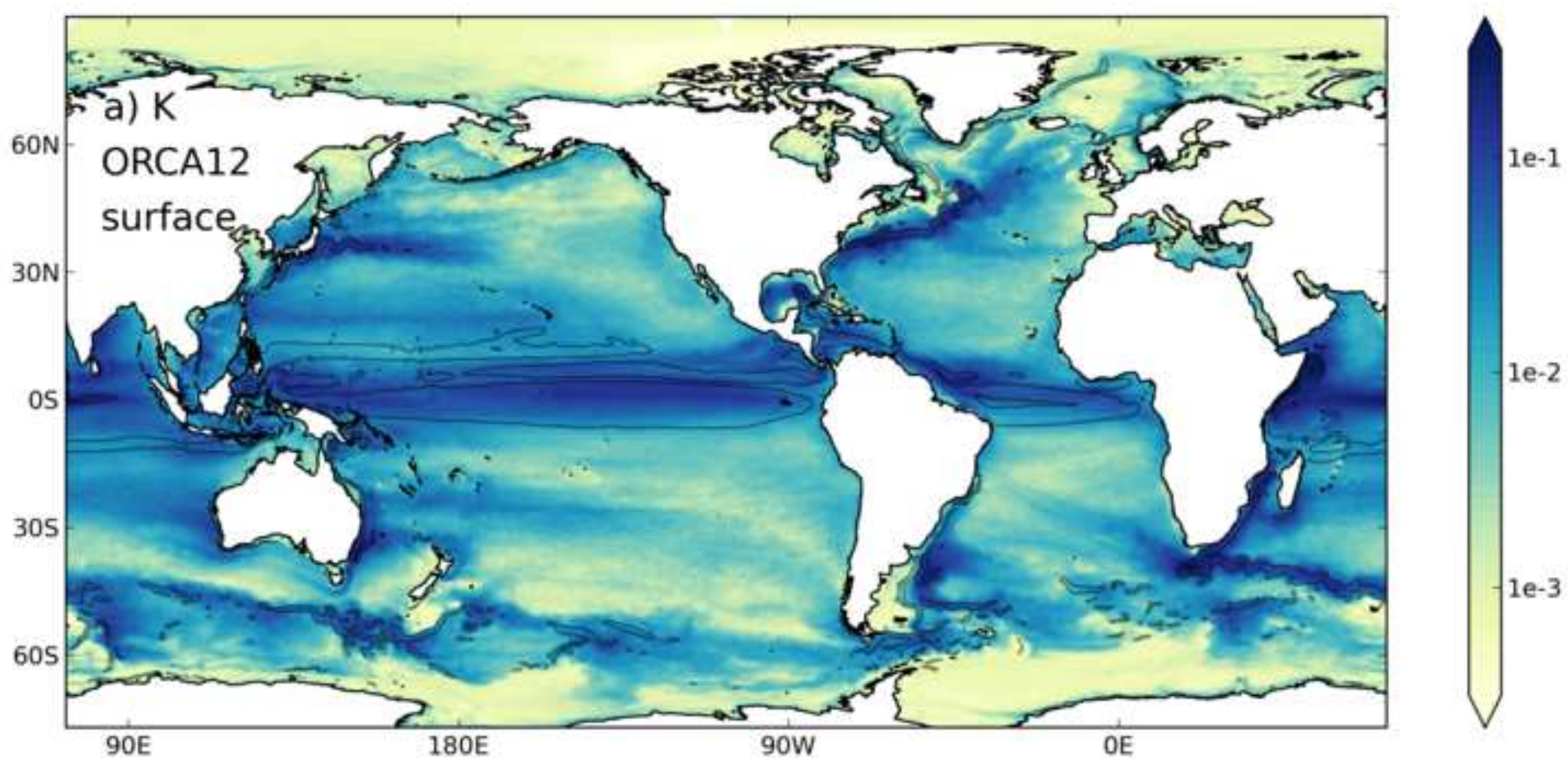


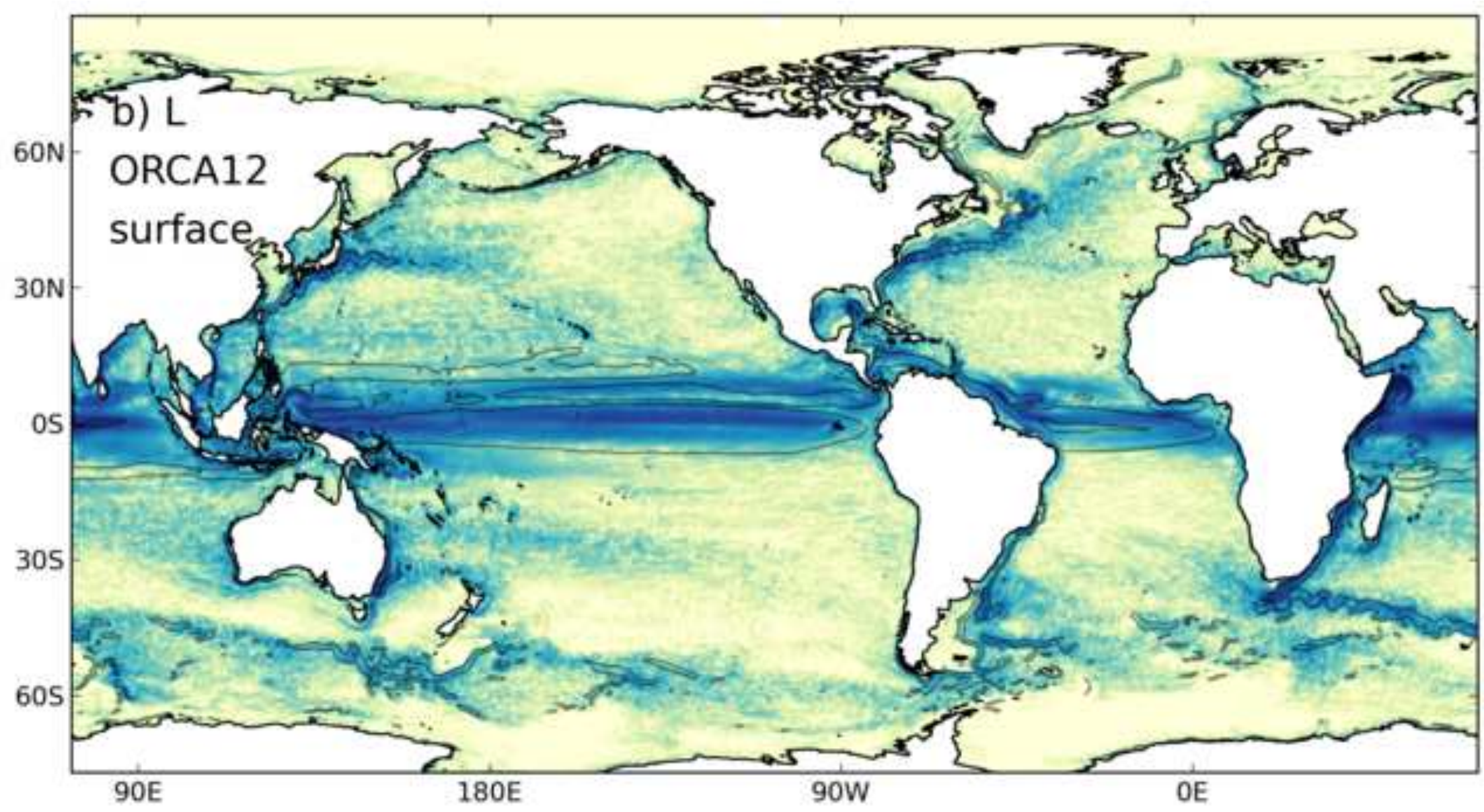

1 e- 1

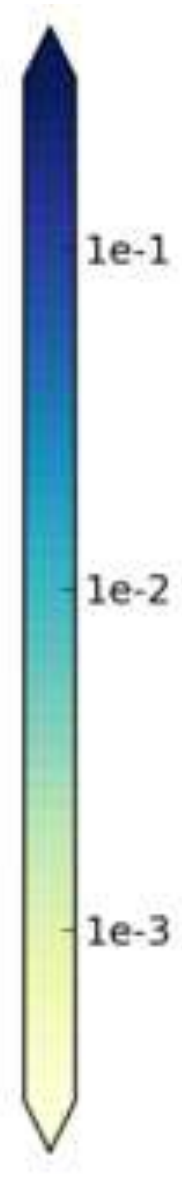



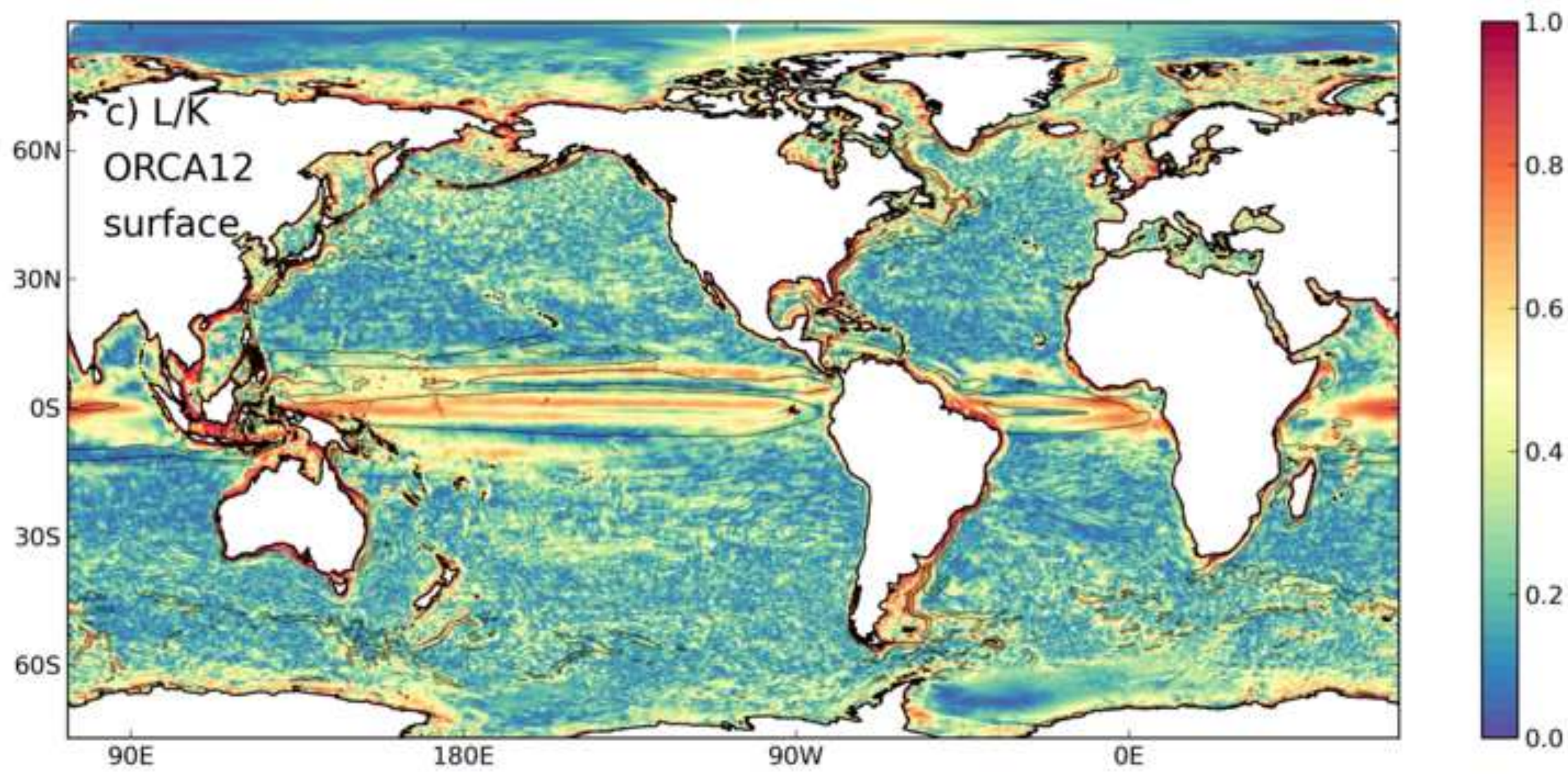

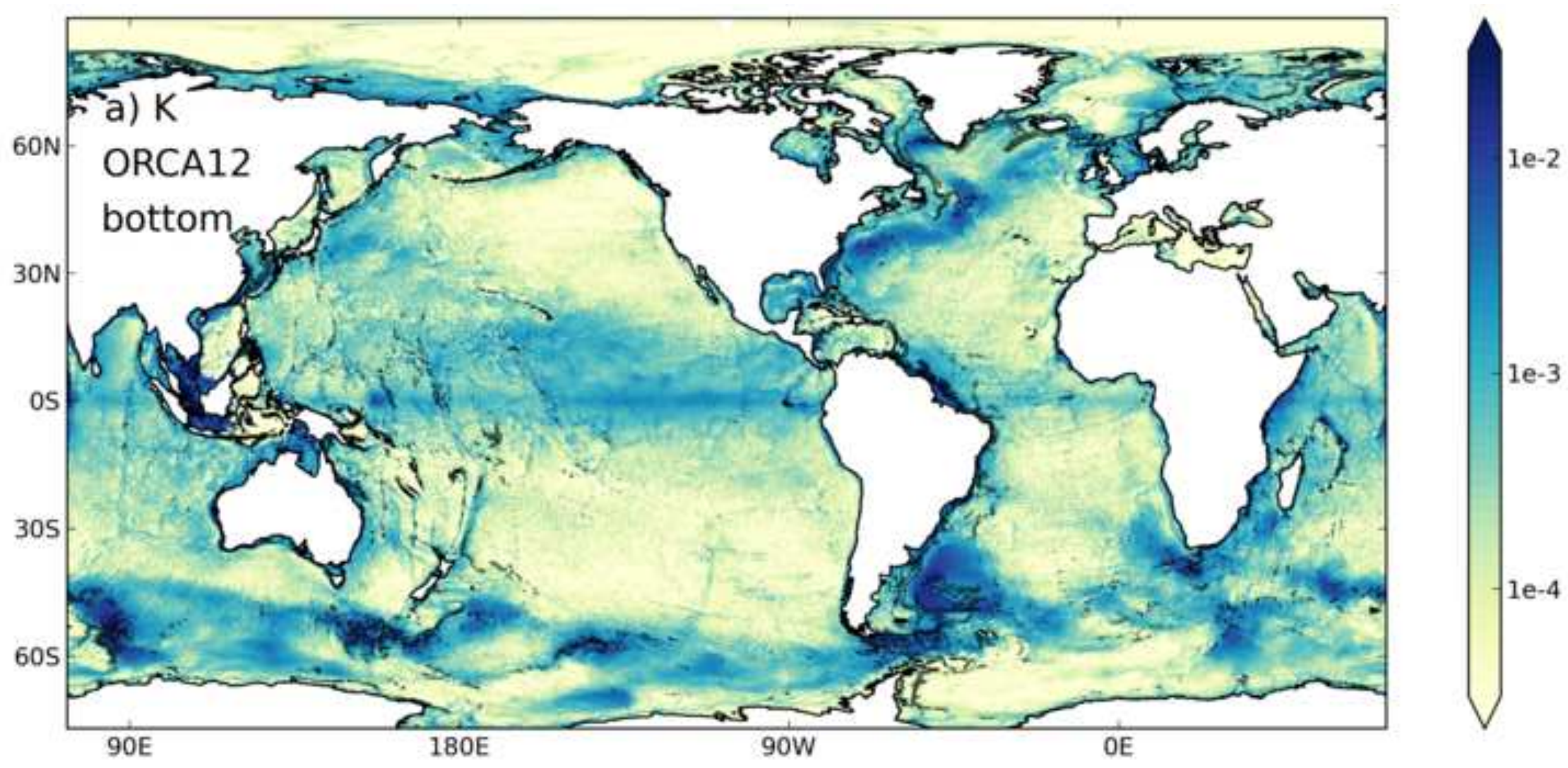


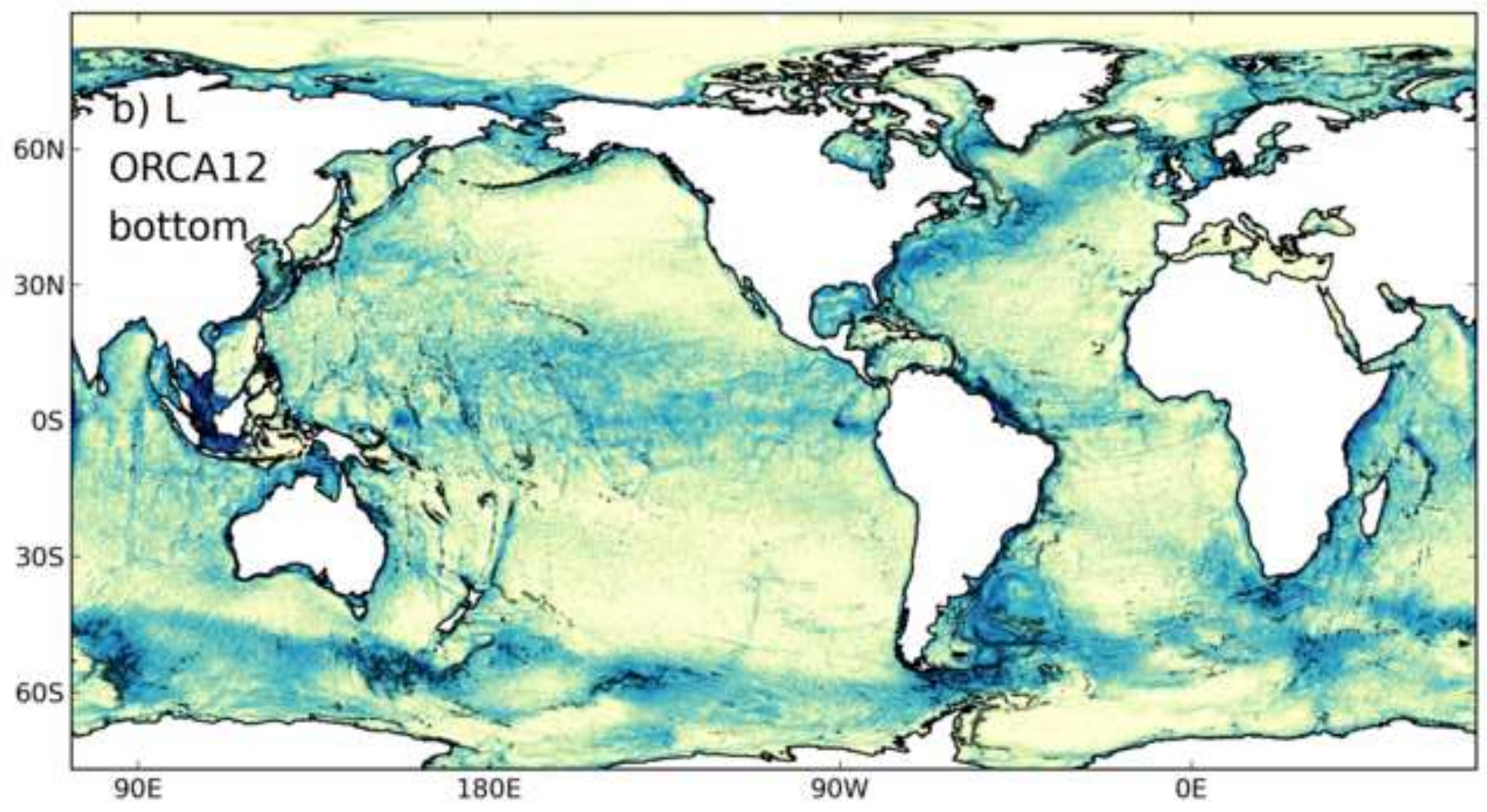



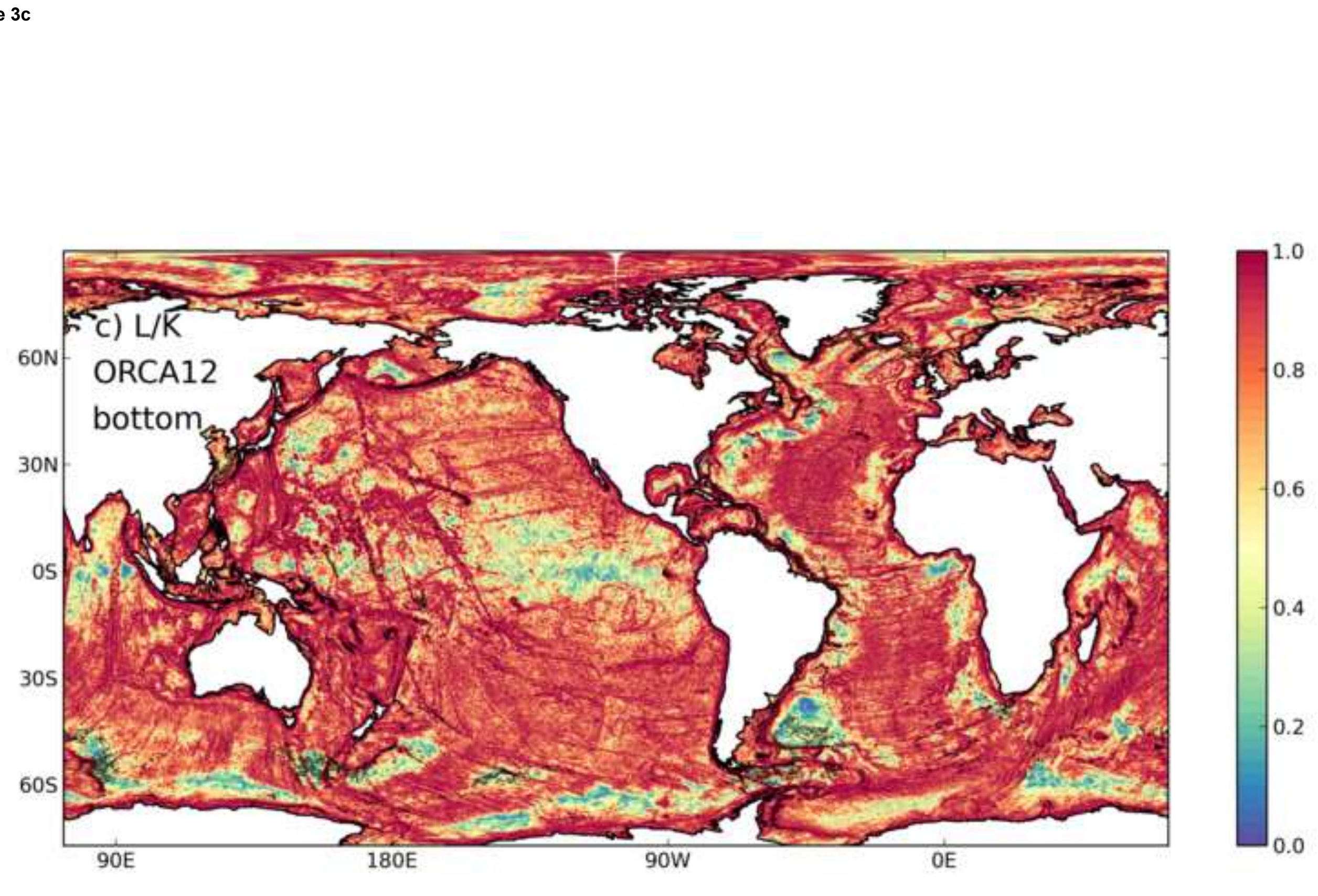

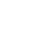

4




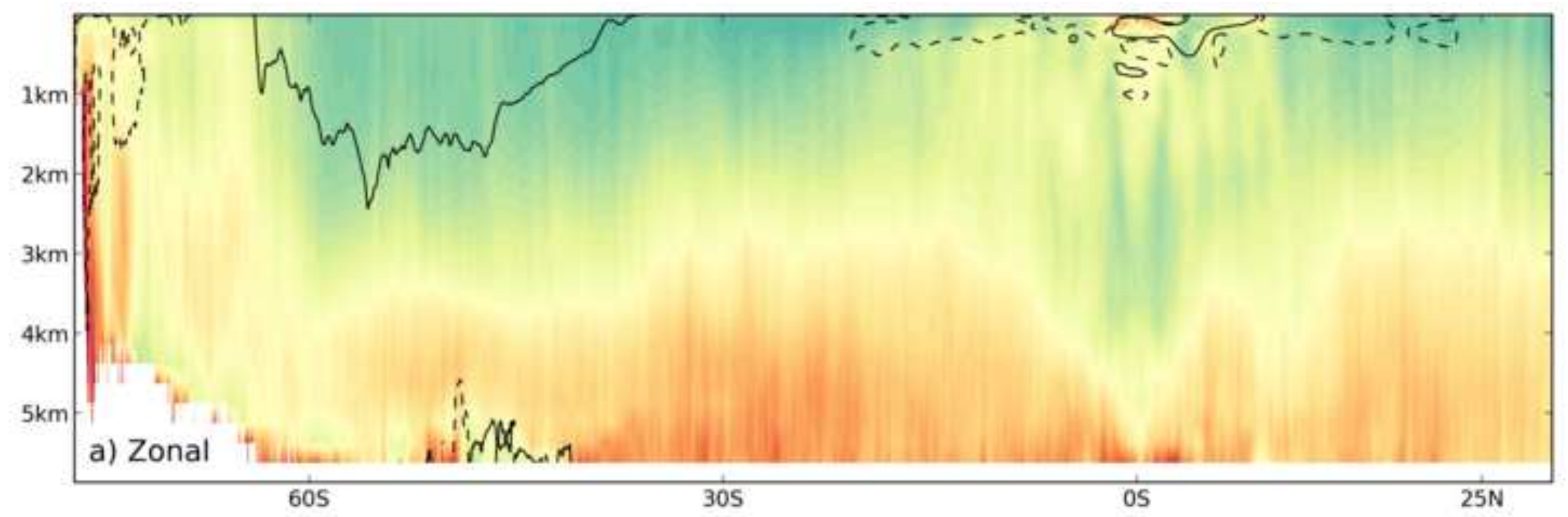


Figure 4b

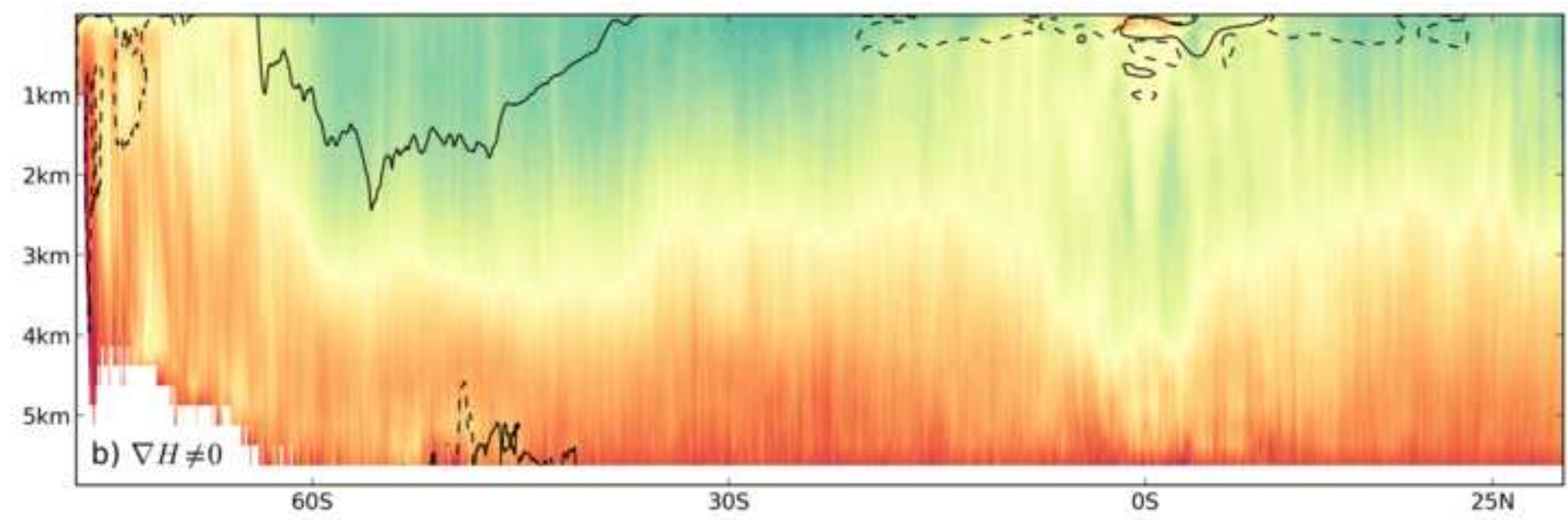




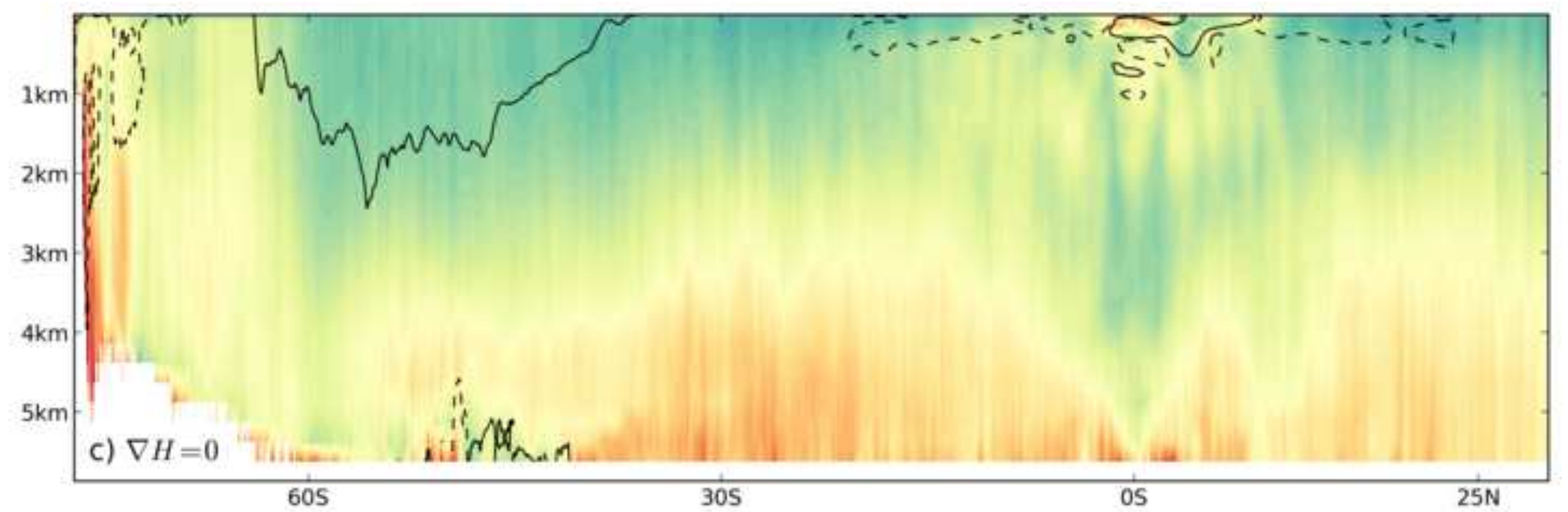




\section{Figure $5 \mathrm{a}$}

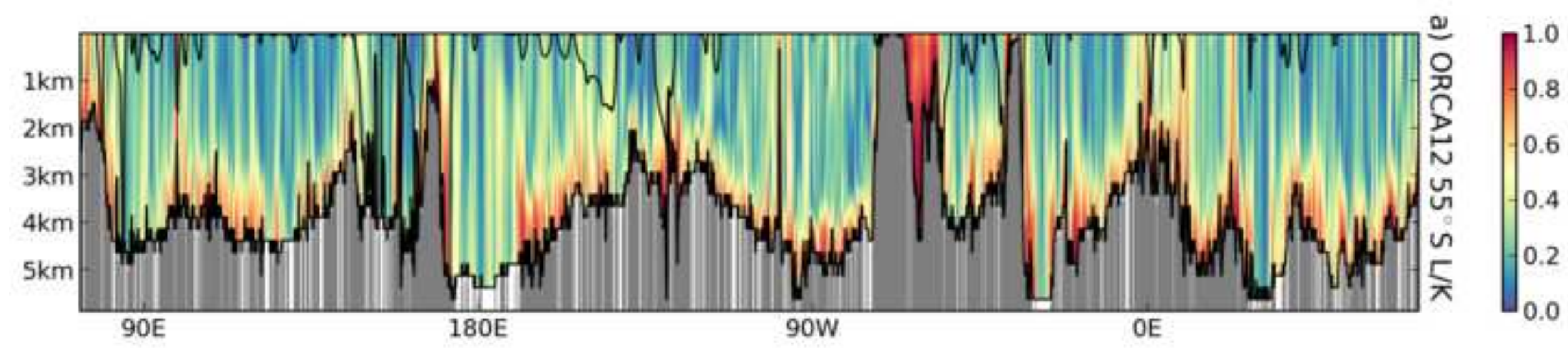




\section{Figure $5 b$}

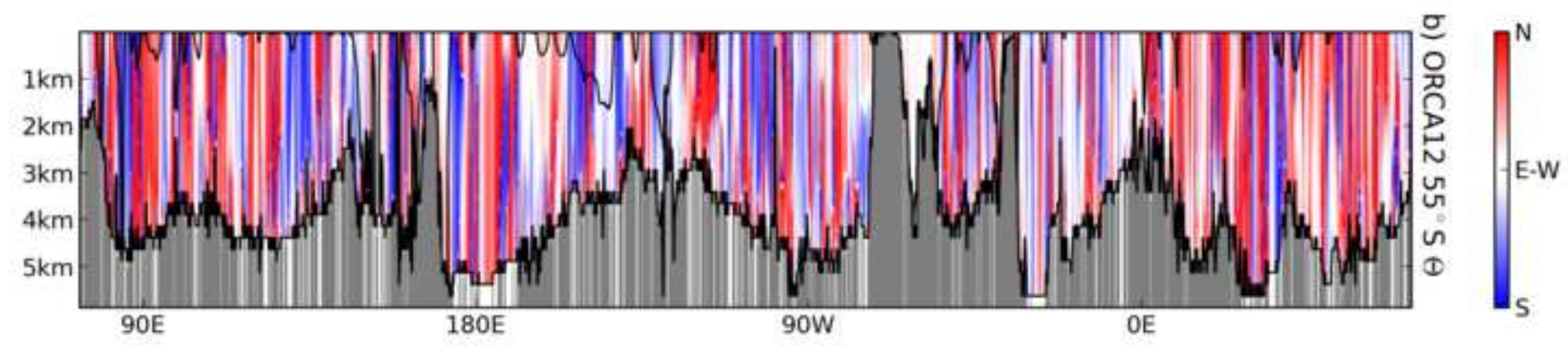




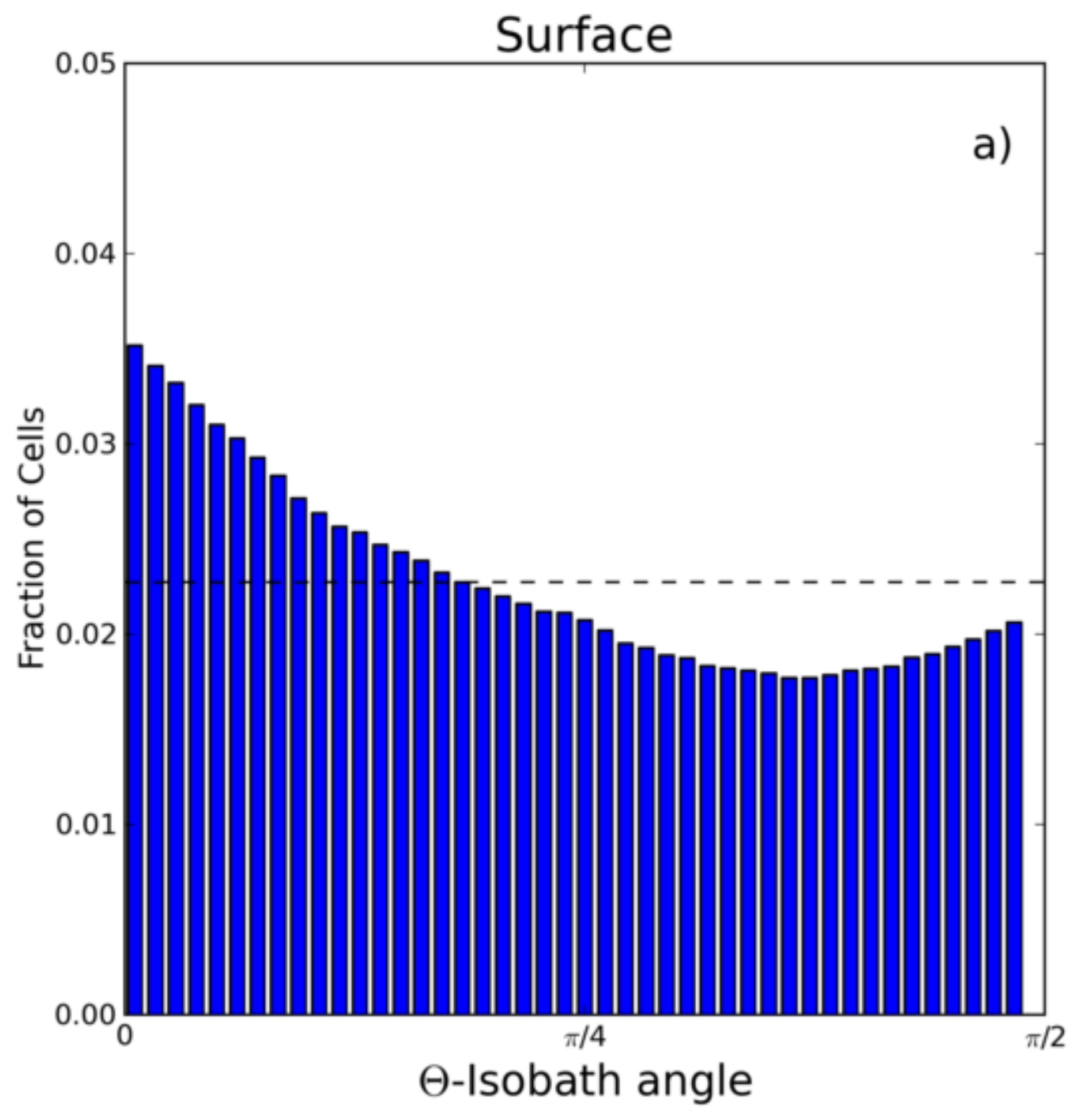

Surface 


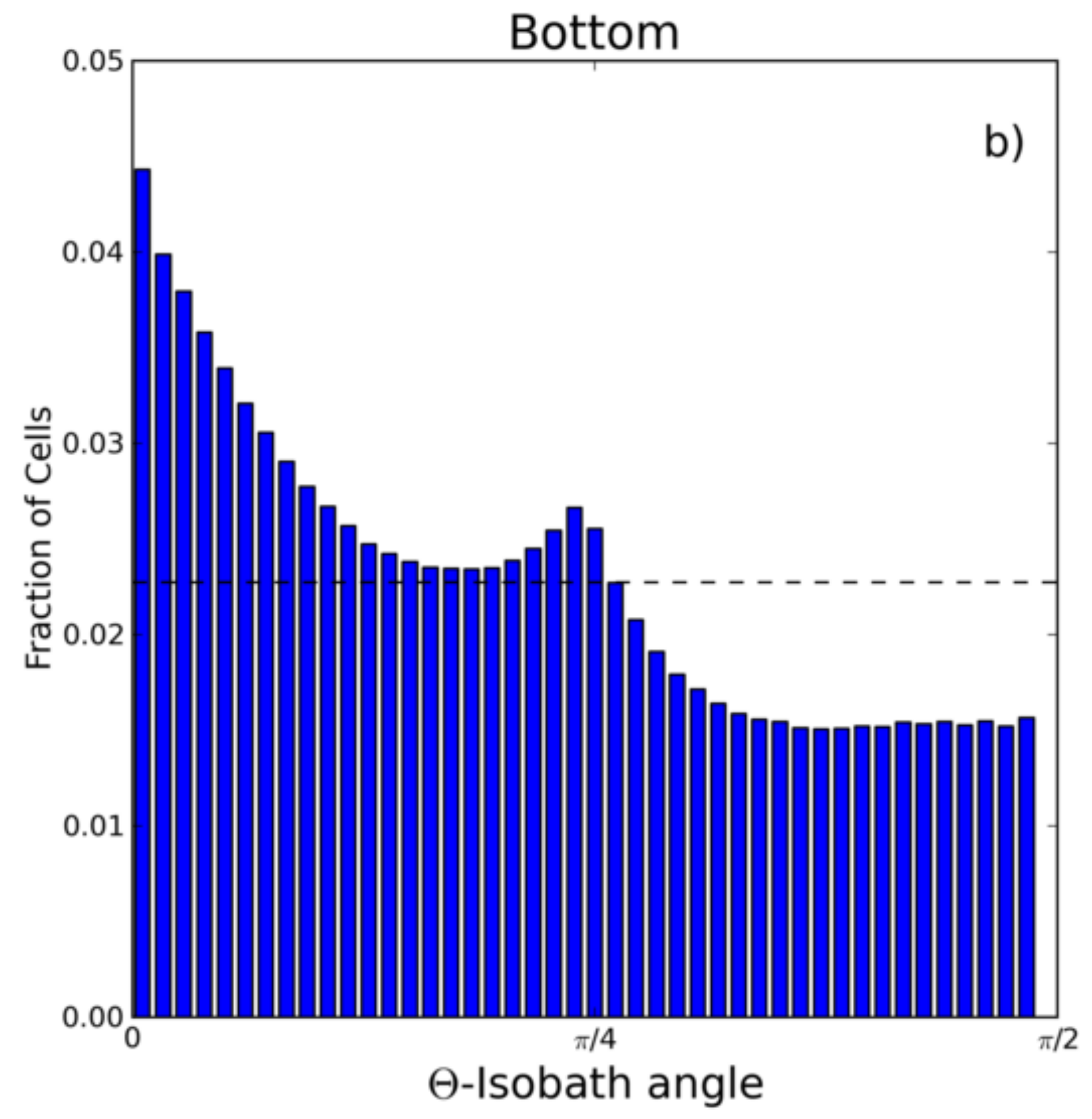




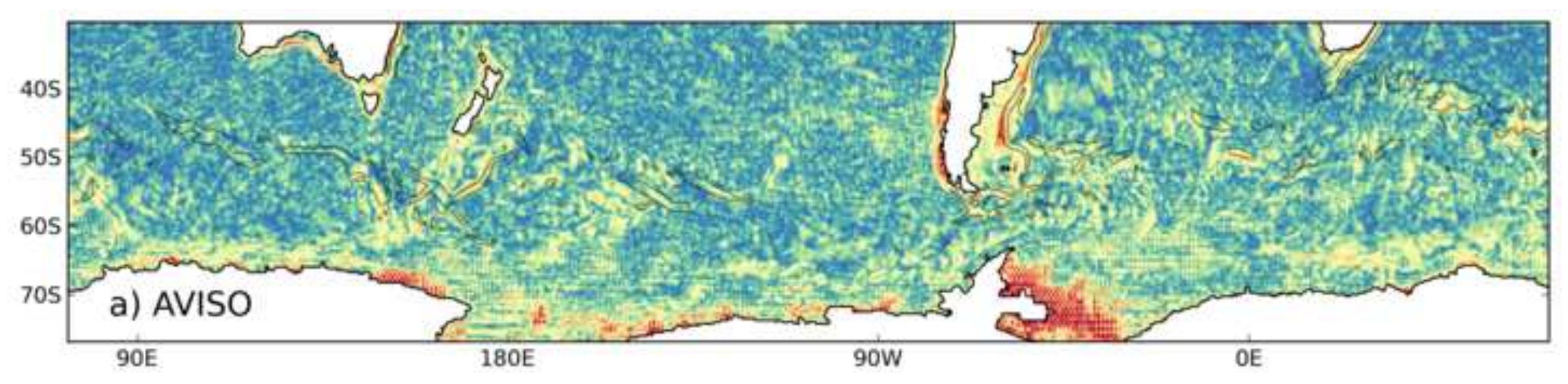

$180 \mathrm{E}$

90W 


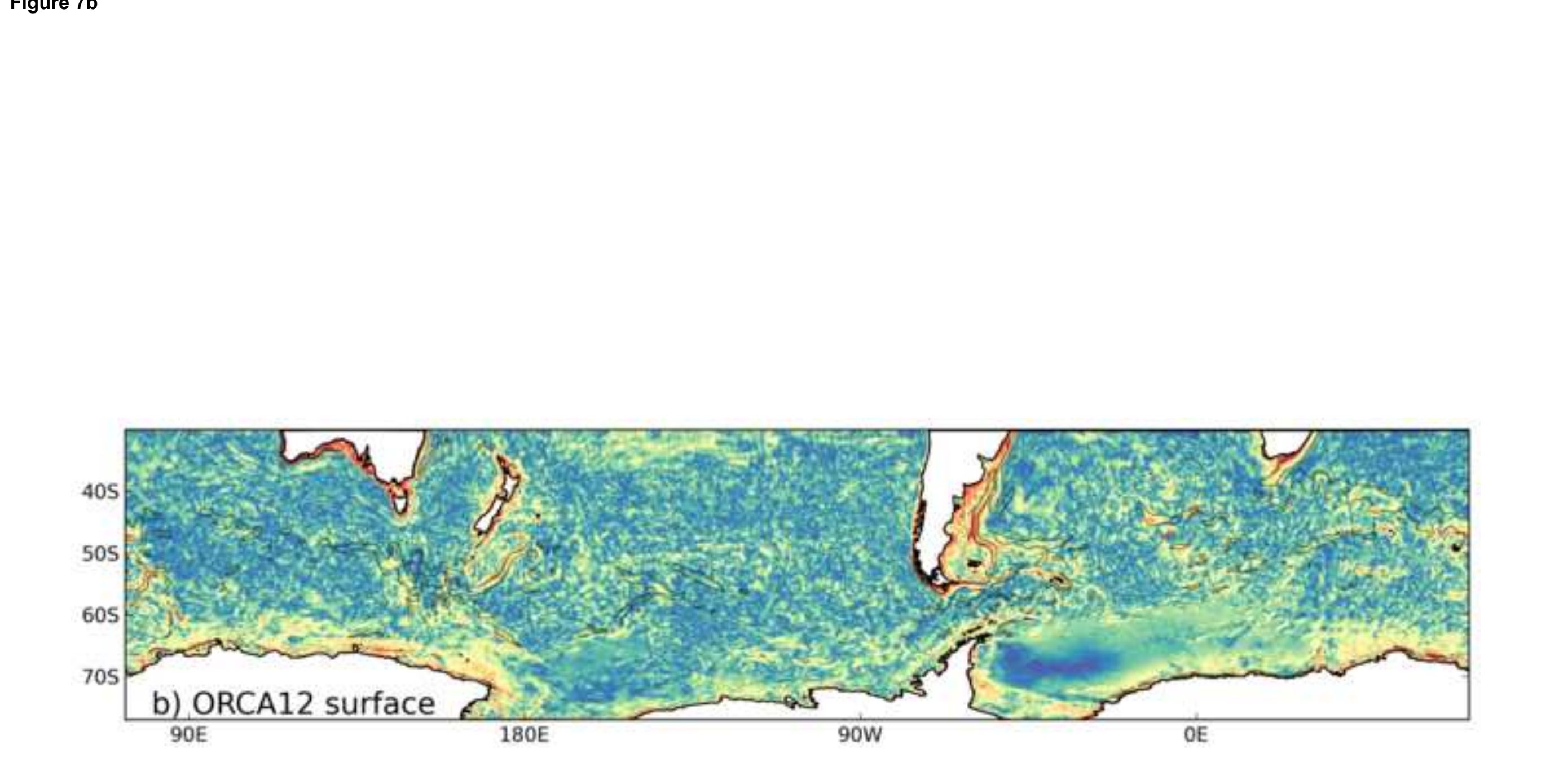

(b
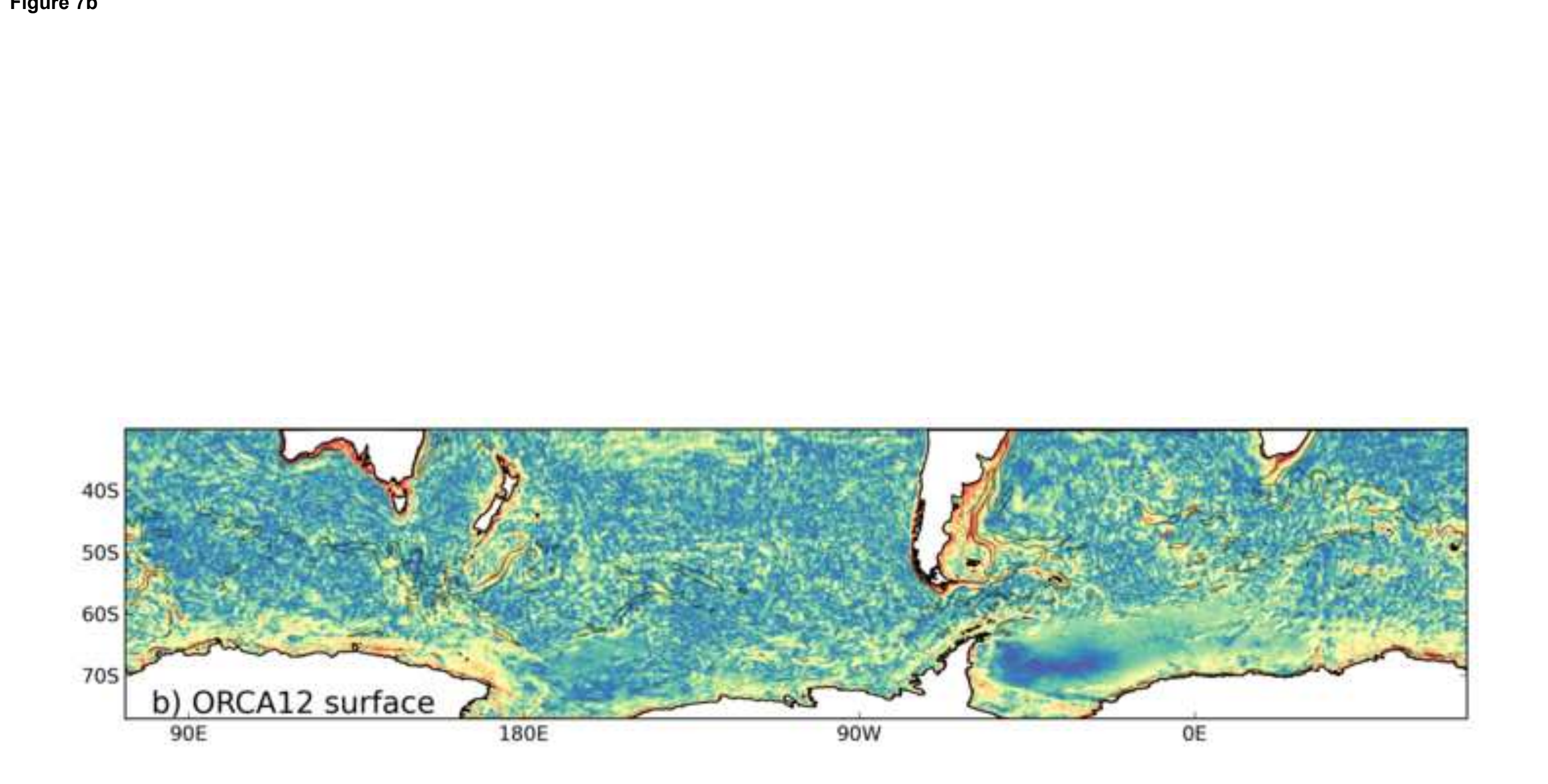


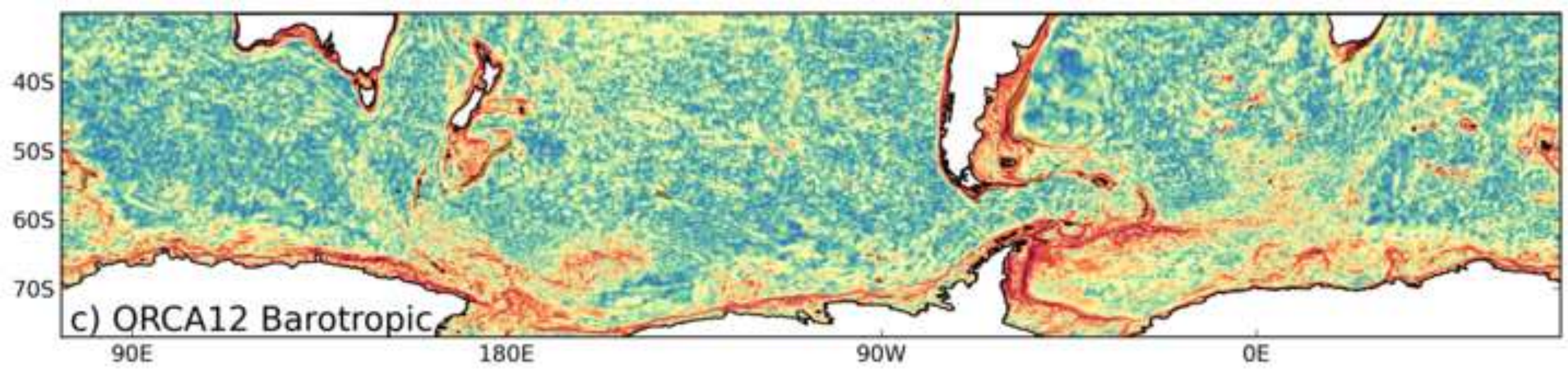




\section{Figure $7 d$

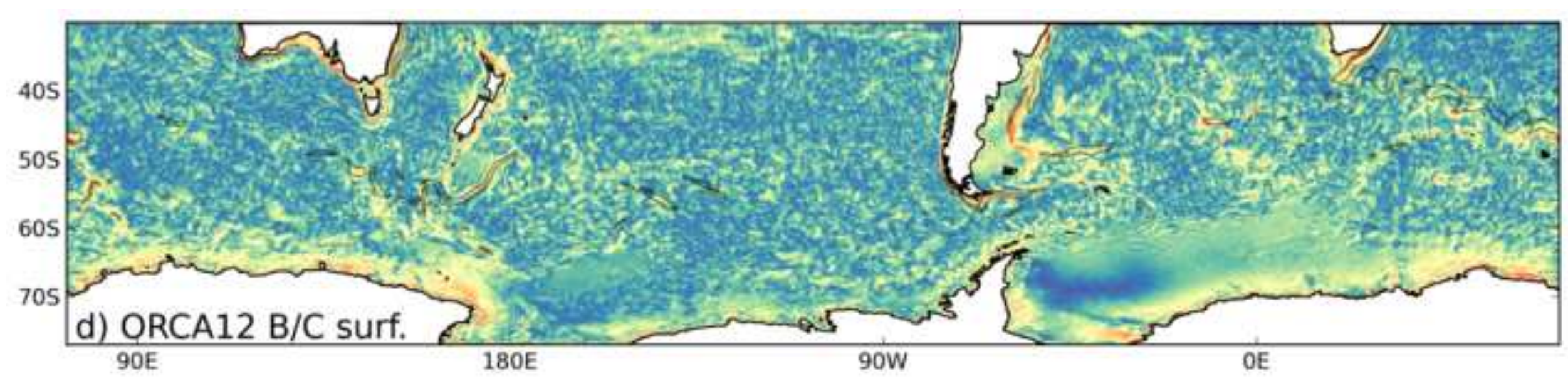




\section{Figure $7 \mathrm{~g}$}

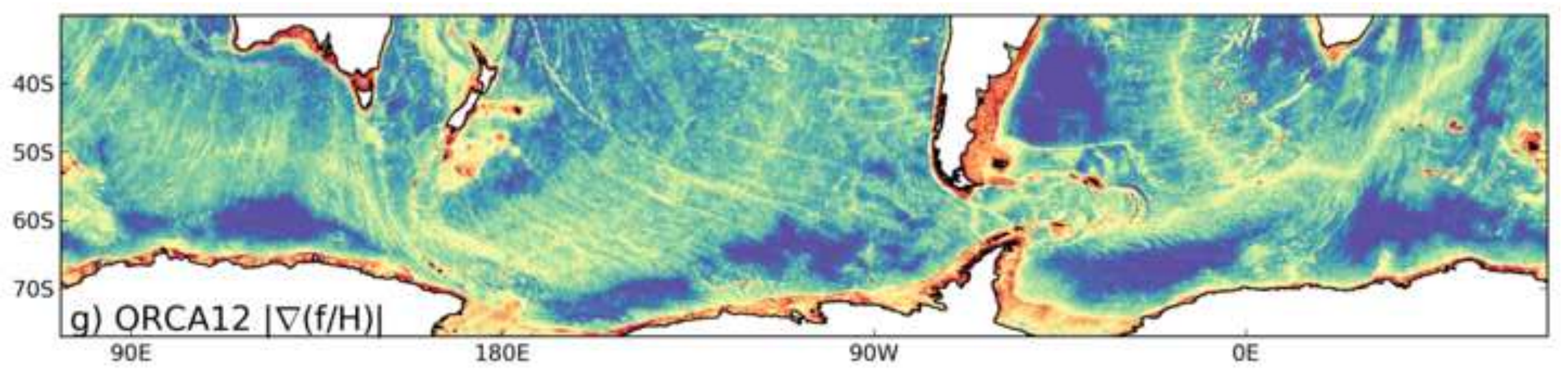


Figure $7 z 1$

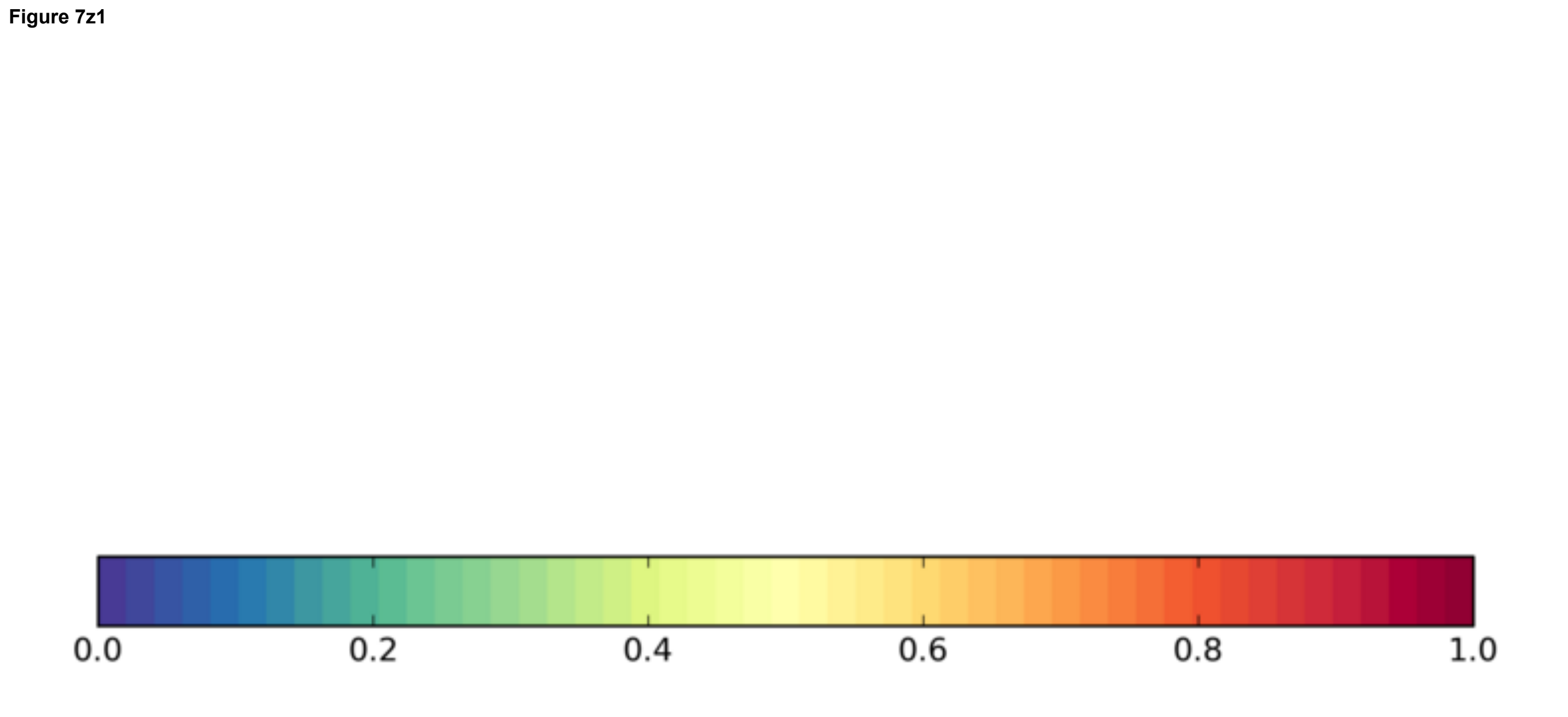

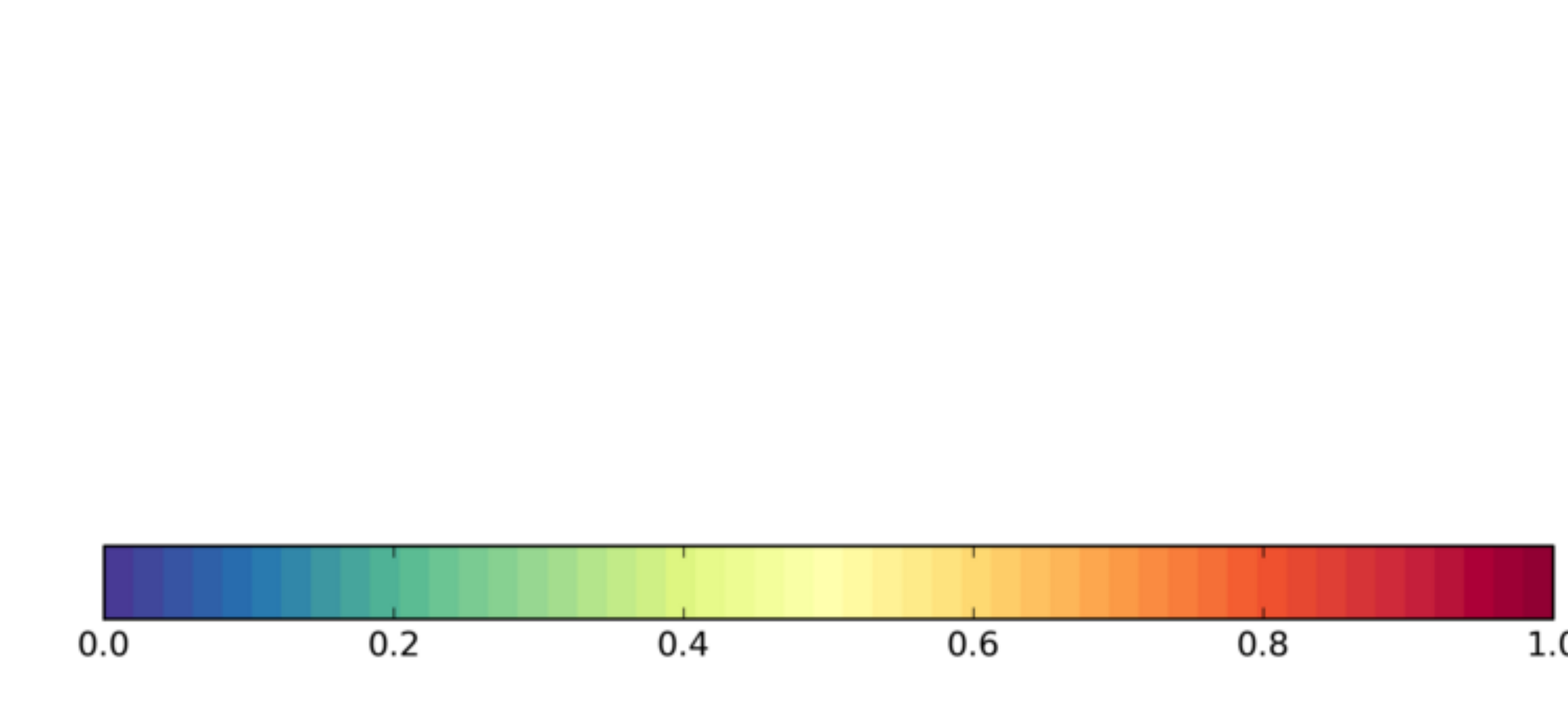

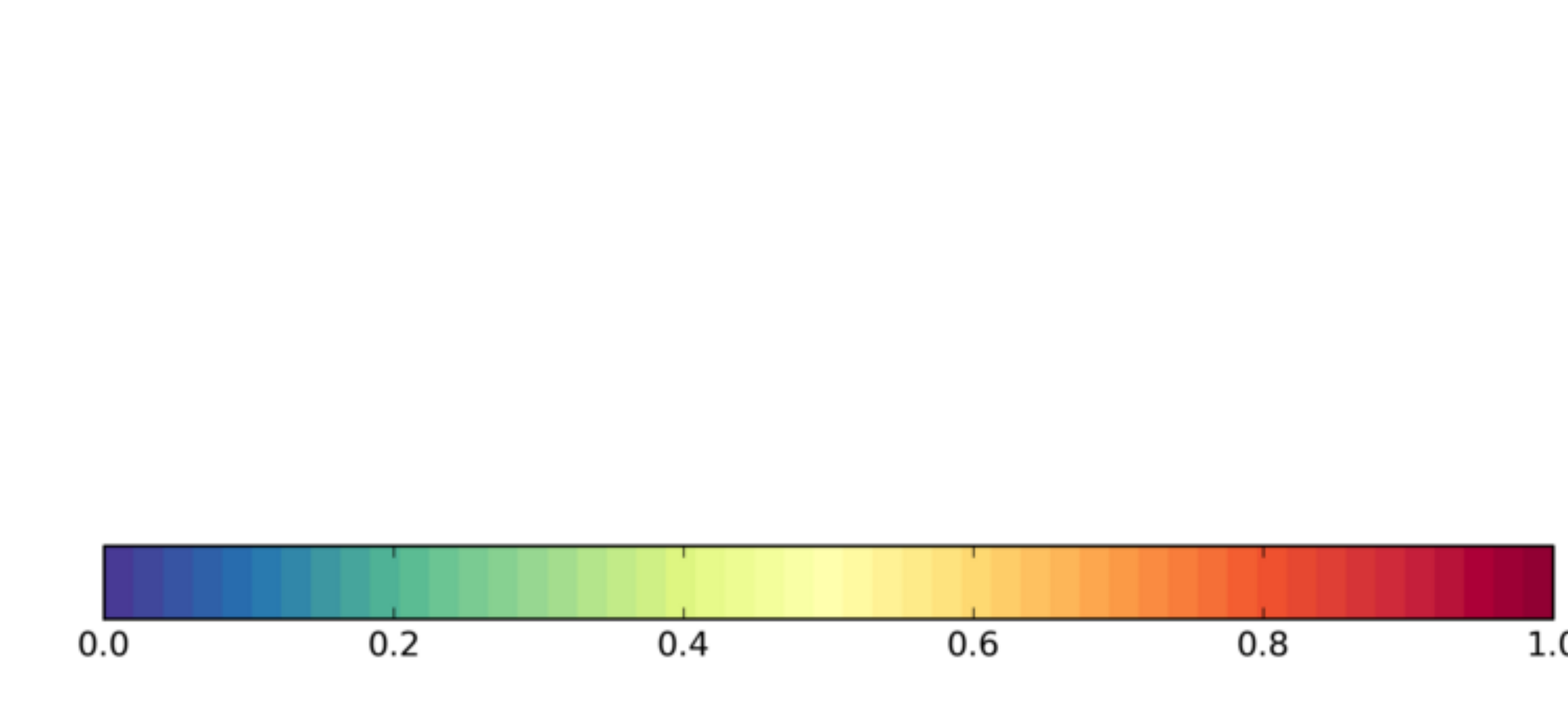

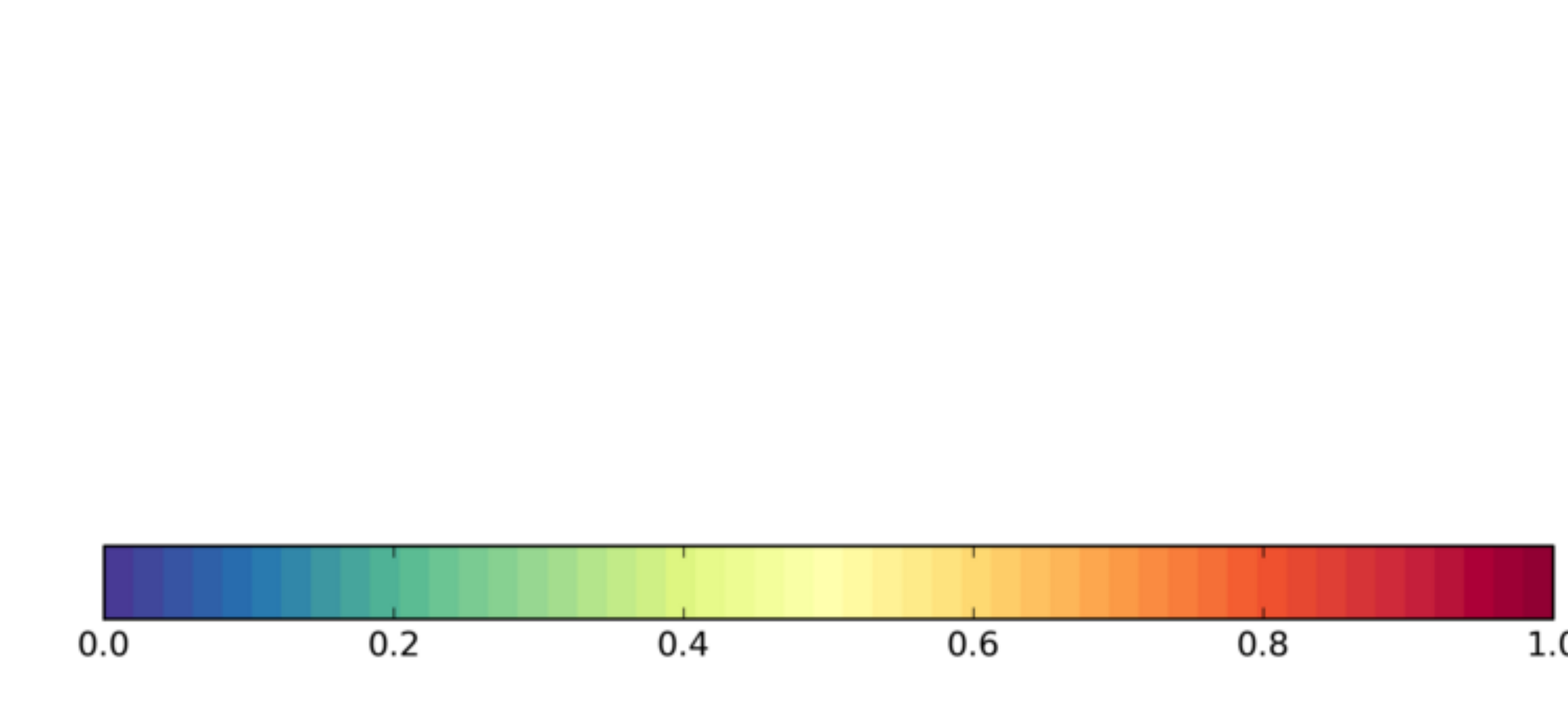

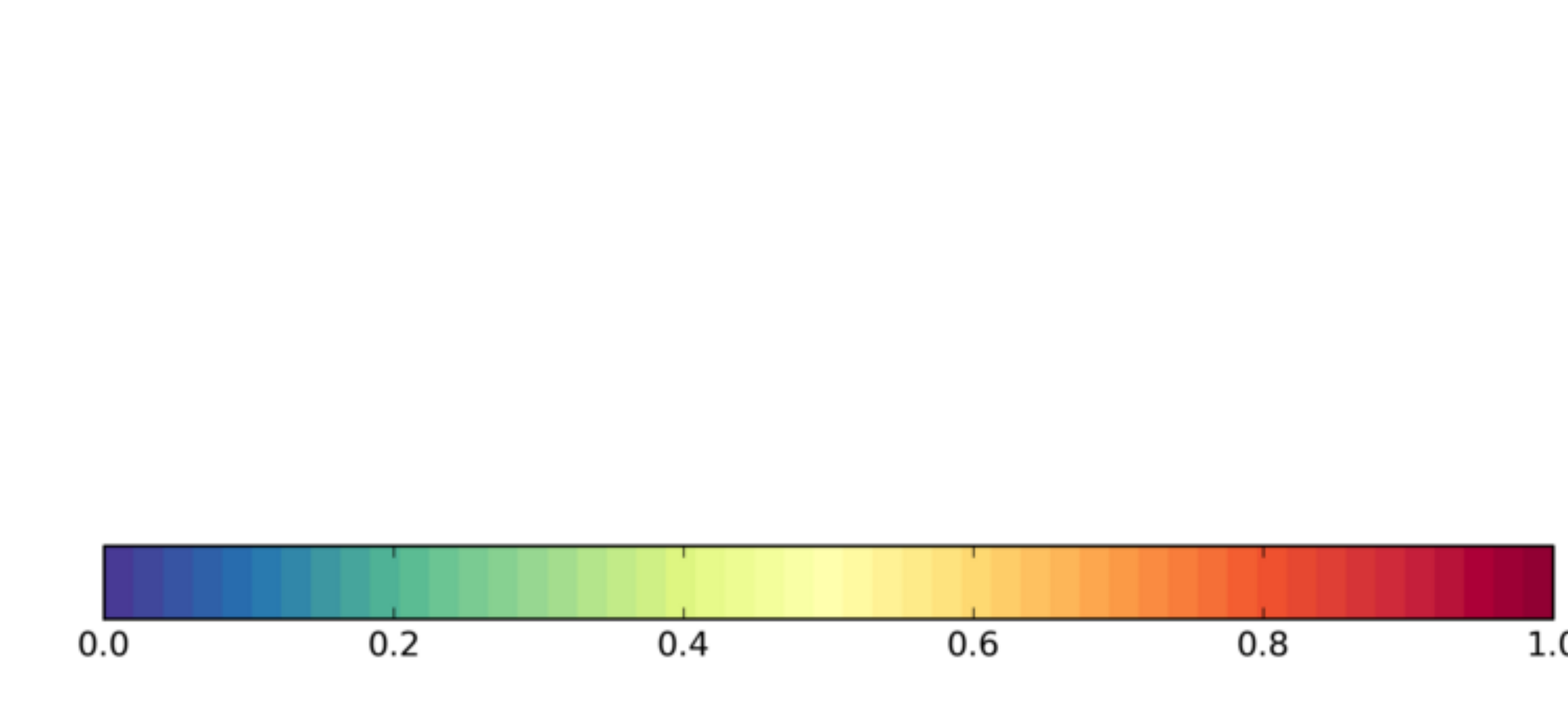
1.0

Figure $7 z$

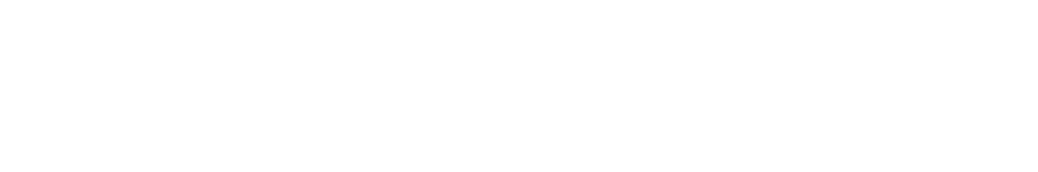

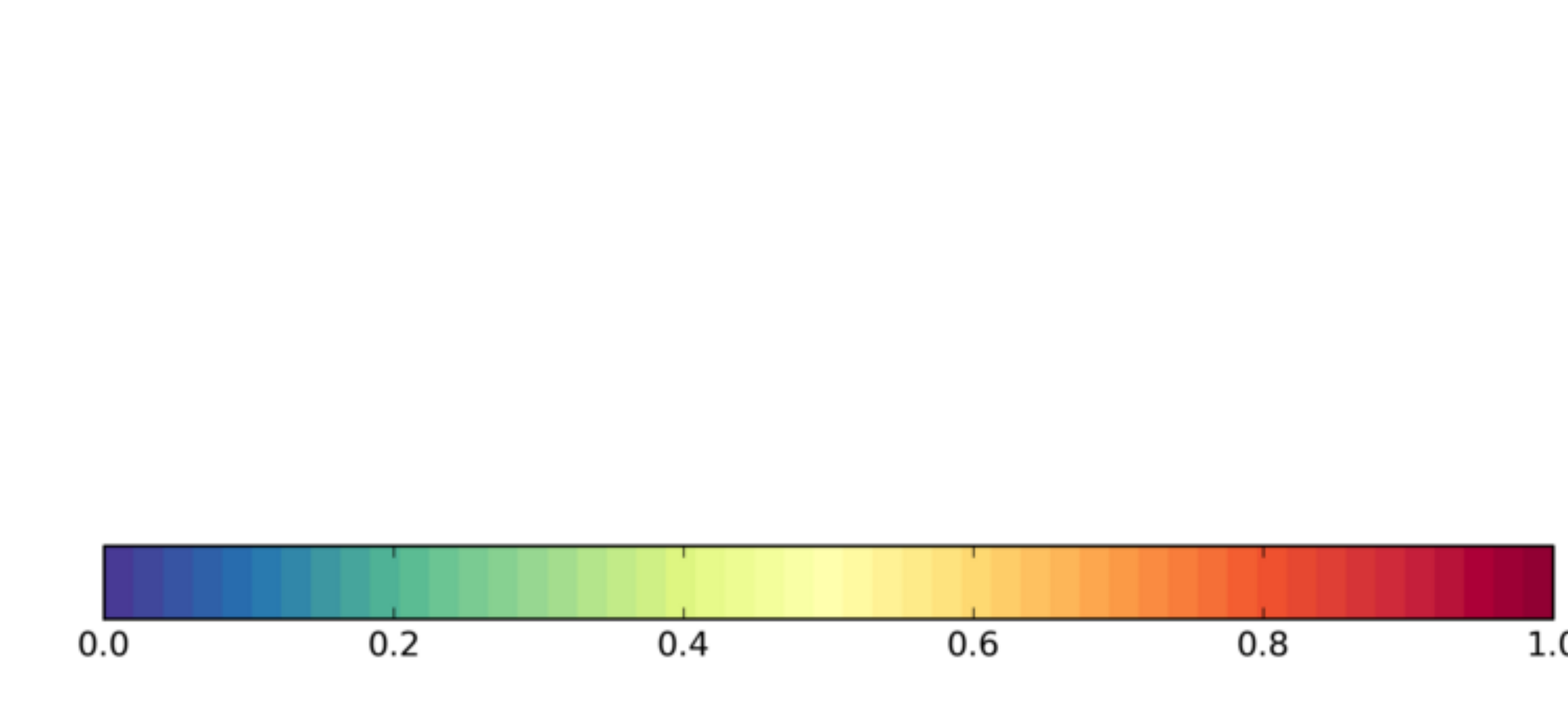

)


$|\nabla(\mathrm{f} / \mathrm{H})|=1 \mathrm{e}-13$

$|\nabla(f / H)|=1 e-12$

$|\nabla(\mathrm{f} / \mathrm{H})|=1 \mathrm{e}-11$

$|\nabla(f / H)|=1 e-10$

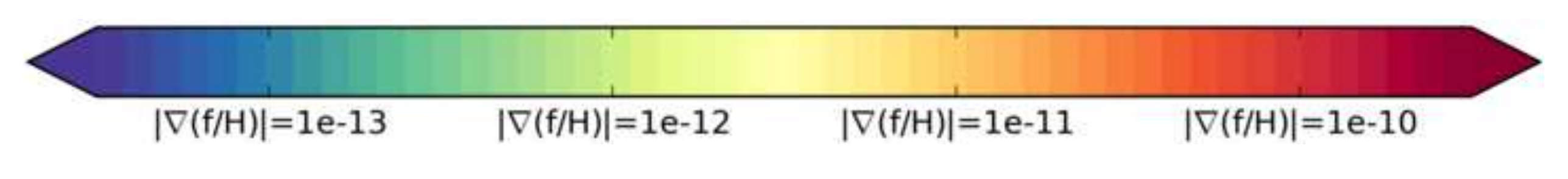




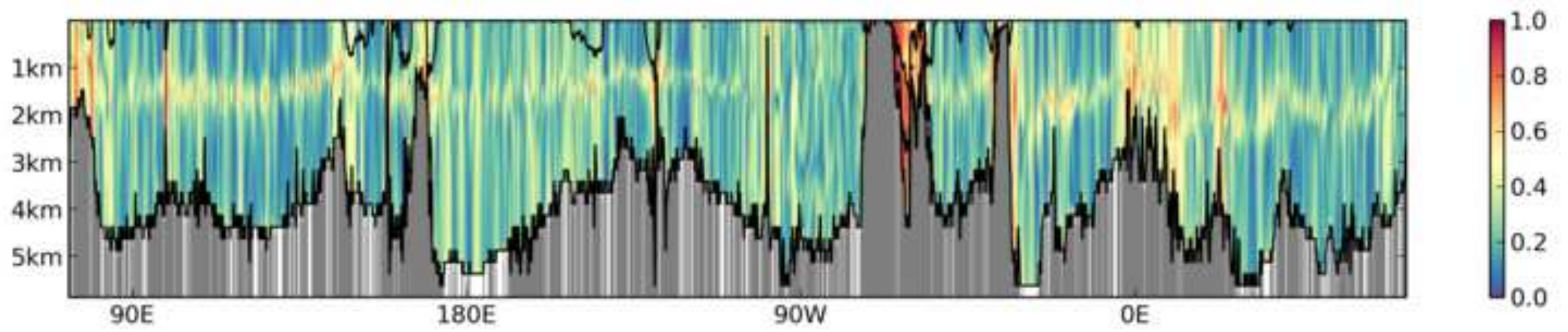



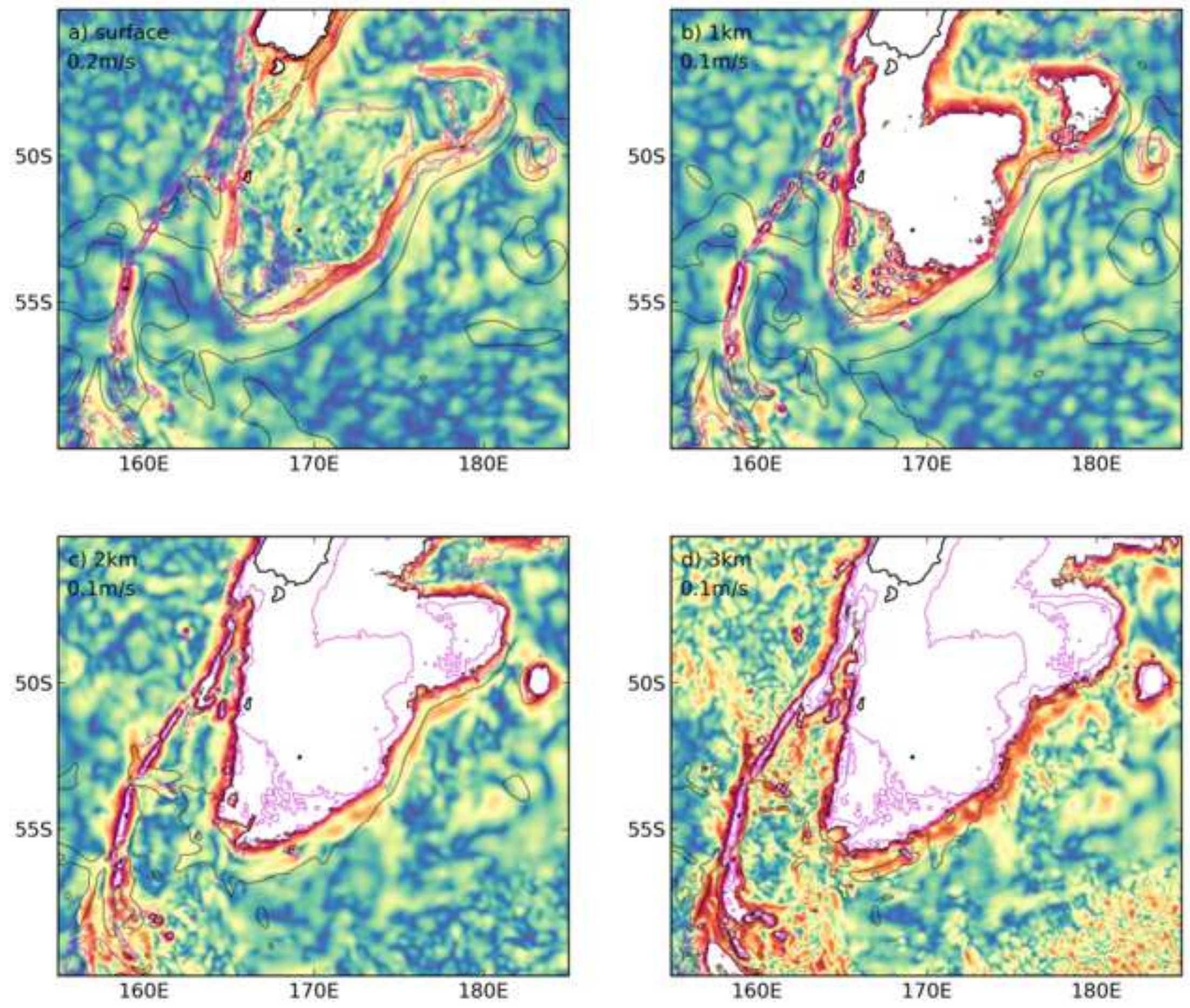
